\title{
Field-induced SU(4) to SU(2) Kondo crossover in a half-filling nanotube dot: Spectral and finite-temperature properties
}

Yoshimichi Teratani, Rui Sakano, Tokuro Hata, Tomonori Arakawa, Meydi Ferrier, Kensuke Kobayashi, and Akira Oguri

\begin{tabular}{|c|l|}
\hline Citation & Physical Review B. 102(16); 165106 \\
\hline Issue Date & 2020-10-06 \\
\hline Type & Journal Article \\
\hline Textversion & Publisher \\
\hline \multirow{2}{*}{ Rights } & $\begin{array}{l}\text { O2020 American Physical Society. This article may be downloaded for personal use } \\
\text { only. Any other use requires prior permission of the author and American Physical } \\
\text { Society. } \\
\text { The following article appeared in Physical Review B Vol.102, Iss.16 and may be } \\
\text { found at https://doi.org/10.1103/PhysRevB.102.165106 . }\end{array}$ \\
\hline DOI & \begin{tabular}{l} 
10.1103/PhysRevB.102.165106 \\
\hline
\end{tabular} \\
\hline
\end{tabular}

\author{
Self-Archiving by Author(s) \\ Placed on: Osaka City University
}

Yoshimichi Teratani, Rui Sakano, Tokuro Hata et al. (2020). Field-induced SU(4) to SU(2) Kondo crossover in a half-filling nanotube dot: Spectral and finite-temperature properties. Physical Review B. 102. doi: 10.1103/PhysRevB.102.165106 


\title{
Field-induced SU(4) to SU(2) Kondo crossover in a half-filling nanotube dot: Spectral and finite-temperature properties
}

\author{
Yoshimichi Teratani $\odot,{ }^{1}$ Rui Sakano, ${ }^{2}$ Tokuro Hata, ${ }^{3}$ Tomonori Arakawa, ${ }^{3,4}$ \\ Meydi Ferrier, ${ }^{3,5}$ Kensuke Kobayashi $\odot,{ }^{3,6}$ and Akira Oguri ${ }^{1,7}$ \\ ${ }^{1}$ Department of Physics, Osaka City University, Sumiyoshi-ku, Osaka, 558-8585, Japan \\ ${ }^{2}$ The Institute for Solid State Physics, the University of Tokyo, Kashiwa, Chiba 277-8581, Japan \\ ${ }^{3}$ Department of Physics, Osaka University, Toyonaka, Osaka 560-0043, Japan \\ ${ }^{4}$ Center for Spintronics Research Network, Osaka University, Toyonaka, Osaka 560-8531, Japan \\ ${ }^{5}$ Laboratoire de Physique des Solides, CNRS, Université Paris-Sud, Université Paris Saclay, 91405 Orsay Cedex, France \\ ${ }^{6}$ Institute for Physics of Intelligence and Department of Physics, The University of Tokyo, Tokyo 113-0033, Japan \\ ${ }^{7}$ Nambu Yoichiro Institute of Theoretical and Experimental Physics, Sumiyoshi-ku, Osaka, 558-8585, Japan
}

(Received 29 March 2020; revised 17 September 2020; accepted 18 September 2020; published 6 October 2020)

\begin{abstract}
We study finite-temperature properties of the Kondo effect in a carbon nanotube (CNT) quantum dot using the Wilson numerical renormalization group (NRG). In the absence of magnetic fields, four degenerate energy levels of the CNT consisting of spin and orbital degrees of freedom give rise to the SU(4) Kondo effect. We revisit the universal scaling behavior of the SU(4) conductance for quarter- and half-filling in a wide temperature range. We find that the filling dependence of the universal scaling behavior at low temperatures $T$ can be explained clearly with an extended Fermi-liquid theory. This theory clarifies that a $T^{2}$ coefficient of conductance becomes zero at quarter-filling, whereas the coefficient at half-filling is finite. We also study a field-induced crossover from the SU(4) to SU(2) Kondo state observed at the half-filled CNT dot. The crossover is caused by the matching of the spin and orbital Zeeman splittings, which lock two levels among the four at the Fermi level even in magnetic fields $B$. We find that the conductance shows the $\mathrm{SU}(4)$ scaling behavior at $\mu_{B} B<k_{B} T_{K}^{\mathrm{SU}(4)}$ and it exhibits the $\mathrm{SU}(2)$ universality at $\mu_{B} B \gg k_{B} T_{K}^{\mathrm{SU}(4)}$, where $T_{K}^{\mathrm{SU}(4)}$ is the $\mathrm{SU}(4)$ Kondo temperature. To clarify how the excited states evolve along the SU(4) to SU(2) crossover, we also calculate the spectral function. The results show that the Kondo resonance width of the two states locked at the Fermi level becomes sharper with increasing fields. The spectral peaks of the other two levels moving away from the Fermi level merge with atomic limit peaks for $\mu_{B} B \gtrsim k_{B} T_{K}^{\mathrm{SU}(4)}$.
\end{abstract}

DOI: 10.1103/PhysRevB.102.165106

\section{INTRODUCTION}

Quantum dots provide an ideal testbed to investigate strong correlations between the electrons in localized levels and the conduction electrons in reservoirs. Kondo effect $[1,2]$ is a typical many-body phenomenon that occurs also in quantum dots having local spin degrees of freedom. The Kondo effect in quantum dots has been studied theoretically $[3,4]$ and experimentally $[5,6]$. In addition to the spin degrees of freedom, carbon nanotube (CNT) quantum dots have also the orbital (valley) degrees of freedom, corresponding to clockwise and counter clockwise orbitals around the nanotube axis [7]. Four energy levels consisting of the spin and orbital degrees of freedom give rise to the $\mathrm{SU}(4)$ Kondo effect in the case where the four localized states are degenerate [8-14]. A number of experiments for nonequilibrium transport have observed the SU(4) Kondo effect [15-20]. Perturbations such as spinorbit coupling $\Delta_{\text {So }}$, valley mixing $\Delta_{K, K^{\prime}}$ and magnetic fields $B$ break the SU(4) symmetry. Effects of such perturbations on the Kondo state are theoretically studied for instance, using Wislon's numerical renormalization group (NRG) approach [21-23], which has been extended to explore transport coefficients and spectral functions with very high accuracy [12,24-26].

The main purpose of the present paper is to clarify the finite temperature properties of the Kondo effect in CNT dots. In the first half of this paper, we study the scaling behavior of the $\mathrm{SU}(4)$ conductance at quarter- and half-filling. Although the scaling behavior has been studied [12,16,27-29], we revisit it with an extended microscopic Fermi-liquid theory which describes transport phenomena at low temperatures [30-34]. It has recently been shown that the $T^{2}$ coefficient $C_{T}$ for the conductance is determined in terms of five Fermi-liquid parameters: electron filling, two linear susceptibilities, and two nonlinear susceptibilities which are defined with respect to the equilibrium ground state. Calculating these parameters using the NRG, we successfully explain the filling dependence of the scaling with the description. Specifically, we explore the filling dependence of a $T^{2}$ coefficient $C_{T}$ and find that $C_{T}$ becomes zero at quarter-filling whereas it is finite at halffilling.

In the second half of this paper, we examine a field induced crossover from $\mathrm{SU}(4)$ to $\mathrm{SU}(2)$ Kondo state at half-filling nanotube dot $[35,36]$. At the valley where the crossover 
occurs, the SU(4) Kondo resonance emerges in the absence of magnetic field, because $\Delta_{K, K^{\prime}}$ and $\Delta_{\text {SO }}$ are smaller than the SU(4) Kondo energy scale, $k_{B} T_{K}^{\mathrm{SU}(4)}$. The field induced crossover is different from the other crossover occurring at quarter-filling [27,28,37-43]. Specifically, this crossover at half-filling occurs in a situation where two localized levels among the four remain the Fermi level even in magnetic fields while the other two levels move away from the Fermi level. The situation realizes if the spin Zeeman splitting coincides the orbital splitting. Such coincidence can reasonably occur in real CNT dots.

In the previous work, we have studied the crossover occurring in this situation, by calculating quasiparticle parameters such as phase shift $\delta$, wave function renormalization factor $Z$ and Wilson ratio $R$ [36]. We have found that the applied magnetic fields enhance the electron correlations. For instance, as magnetic fields increase, the renormalization factor $Z$ decreases from the $\mathrm{SU}(4)$ value to the $\mathrm{SU}(2)$ value and thus the Kondo energy scale $T_{K}$ decreases. Our NRG results are in good agreement with the experimental observations [35].

In this paper, we calculate the temperature dependence of the conductance in magnetic fields to clarify the crossover in a wide range of temperature $T$. We show that a temperature scale $T^{*}$ around which the conductance shows $\log T$ dependence decreases with increasing magnetic fields. This decrease of $T^{*}$ becomes clearer in a strong Coulomb interaction case and agree with the field dependence of $Z$. We also examine the scaling behavior of the conductance. Whereas the conductance follows the $\mathrm{SU}(4)$ scaling at $\mu_{B} B<k_{B} T_{K}^{\mathrm{SU}(4)}$, it shows the $\mathrm{SU}(2)$ scaling at $\mu_{B} B \gg k_{B} T_{K}^{\mathrm{SU}(4)}$.

In addition to the conductance, we calculate the level resolved spectral functions in magnetic fields. The component for the doubly degenerate levels shows that the Kondo resonance width becomes sharper with increasing magnetic fields. This field dependence of the width corresponds to that of $T^{*}$. Spectral weights of the other two states transfer from the Fermi level, and the peaks merge with atomic limit peaks.

This paper is organized as follows. In the next section, we describe the microscopic Fermi-liquid theory and the NRG approach to CNT dots. In Sec. III, we examine the scaling behavior of the SU(4) conductance at quarter- and half-filling. We discuss how the quasiparticle parameters evolve during the field induced crossover in Sec. IV. We present the NRG results of conductance and discuss the influence of magnetic fields on the temperature dependence of conductance in Sec. V. The spectral functions in increasing magnetic fields are shown in Sec. VI. Summary is given in Sec. VII.

\section{FORMULATION}

Transport properties of carbon nanotube quantum dots are determined by a linear combination of four one-particle levels, consisting of the spin $(\uparrow, \downarrow)$ and valley $\left(K, K^{\prime}\right)$ degrees of freedom. The structure of these four states staying near the Fermi level depend on the inter-valley scattering, the spinorbit coupling, and the Zeeman splittings of the spin and orbital degrees of freedom. We introduce the Anderson model for the CNT dot in this section using a diagonal form for these local levels. We provide a more microscopic description specific to a real CNT dot, in which experiments have measured the current and corresponding noise with high accuracy. The renormalized parameters that characterize the low-energy Fermi-liquid properties and details of the NRG calculations are also described in this section.

\section{A. Anderson model for CNT quantum dots}

We start with an Anderson impurity model for a CNT dot, which has $N=4$ internal degrees of freedom and is connected to two noninteracting leads:

$$
\begin{gathered}
\mathcal{H}=\mathcal{H}_{d}^{0}+\mathcal{H}_{U}+\mathcal{H}_{c}+\mathcal{H}_{T}, \\
\mathcal{H}_{d}^{0}=\sum_{m=1}^{N} \epsilon_{m} d_{m}^{\dagger} d_{m}, \quad \mathcal{H}_{U}=U \sum_{m<m^{\prime}} n_{d m} n_{d m^{\prime}}, \\
\mathcal{H}_{c}=\sum_{\nu=L, R} \sum_{m=1}^{N} \int_{-D}^{D} d \varepsilon \varepsilon c_{\nu, \varepsilon m}^{\dagger} c_{\nu, \varepsilon m}, \\
\mathcal{H}_{T}=\sum_{\nu=L, R} \sum_{m=1}^{N} v_{\nu}\left(\psi_{\nu, m}^{\dagger} d_{m}+d_{m}^{\dagger} \psi_{\nu, m}\right), \\
\psi_{\nu, m} \equiv \int_{-D}^{D} d \varepsilon \sqrt{\rho_{c}} c_{v, \varepsilon, m}, \quad n_{d m} \equiv d_{m}^{\dagger} d_{m} .
\end{gathered}
$$

Here, $d_{m}^{\dagger}$ and $d_{m}$ are the creation and annihilation operators, respectively, for an electron with energy $\varepsilon_{m}$ in the $m$ th discrete one-particle eigenstate of the $\operatorname{dot}(m=1,2, \ldots, N)$. We shall also call $m$ the "flavour" in the following. The Coulomb interactions $U$ between the electrons occupying the dot levels are assumed to be independent of $m$, assuming that the intraand intervalley Coulomb repulsions to be identical. We also assume that Hund's rule coupling can be neglected. This is consistent with the CNT dot in which the field-induced SU(4) to $\mathrm{SU}(2)$ Kondo crossover has been observed [19], and also with the other nanotube dots [15]. $\mathcal{H}_{c}$ describes conduction bands in the leads on the left and right. $v=L$ and $v=R$ respectively denote the left and right leads. The conduction electrons are assumed to carry the flavour index $m$, and the continuous energy states in the bands are normalized such that $\left\{c_{v, \varepsilon m}, c_{v^{\prime}, \varepsilon^{\prime} m^{\prime}}^{\dagger}\right\}=\delta\left(\varepsilon-\varepsilon^{\prime}\right) \delta_{v v^{\prime}} \delta_{m m^{\prime}}$. The Fermi level is chosen to be $\varepsilon_{F}=0$, at the center of the flat conduction bands with the width $2 D . \mathcal{H}_{T}$ describes charge transfer between the dot and leads. We assume that the tunneling matrix element $v_{v}$ is independent of flavour $m$, which can also be justified for a class of CNT dots $[19,35]$. In this situation, the resonance width due to these hybridizations becomes $\Delta=\Delta_{L}+\Delta_{R}$ with $\Delta_{v} \equiv \pi \rho_{c} v_{v}^{2}$, and $\rho_{c}=1 / 2 D$ the density of states of the conduction band. Unless otherwise stated, we set the Boltzmann constant $k_{B}$ to unity, i.e., $k_{B}=1$.

This Hamiltonian $\mathcal{H}$ has the $\mathrm{SU}(N)$ symmetry when all the impurity energies are equal $\epsilon_{m} \equiv \epsilon_{d}$. This is because $\mathcal{H}_{T}$ that describes charge transfer preserves the flavour index $m$, and the Coulomb interaction is determined essentially by the total number of impurities electrons $\widehat{N}_{d} \equiv \sum_{m} n_{d m}$ as $\mathcal{H}_{U}=$ $(U / 2) \widehat{N}_{d}\left(\widehat{N}_{d}-1\right)$. In Appendix A, we explain the symmetry of this Hamiltonian in more detail. 


\section{B. Fermi-liquid parameters}

The equilibrium retarded Green $G_{m}^{r}(\omega)$ is an useful tool to study transport coefficients of quantum dots. $G_{m}^{r}(\omega)$ is given by

$$
\begin{aligned}
& G_{m}^{r}(\omega, T) \equiv-i \int_{0}^{\infty} d t e^{i\left(\omega+i 0^{+}\right) t}\left\langle\left\{d_{m}(t), d_{m}^{\dagger}(0)\right\}\right\rangle \\
&=\frac{1}{\omega-\epsilon_{m}+i \Delta-\Sigma_{m}(\omega, T)} \\
& A_{m}(\omega, T) \equiv-\frac{1}{\pi} \operatorname{Im} G_{m}^{r}(\omega, T) .
\end{aligned}
$$

Here, $\langle\mathcal{O}\rangle \equiv \operatorname{Tr}\left[\mathcal{O} e^{-\mathcal{H} / T}\right] / \Xi$ with $\Xi \equiv \operatorname{Tr} e^{-\mathcal{H} / T}$ is the thermal average of an observable $\mathcal{O}$.

The phase shift $\delta_{m}$ is a primary parameter that characterizes the Fermi-liquid ground state. It is determined by the value of the self-energy $\Sigma_{m}(\omega, T)$ at $\omega=T=0$,

$$
\delta_{m}=\frac{\pi}{2}-\tan ^{-1}\left[\frac{\epsilon_{m}+\Sigma_{m}(0,0)}{\Delta}\right] .
$$

The Friedel sum rule also relates the phase shift $\delta_{m}$ to the occupation number $\left\langle n_{d m}\right\rangle$ [44],

$$
\left\langle n_{d m}\right\rangle=\frac{\partial \Omega}{\partial \epsilon_{m}} \stackrel{T \rightarrow 0}{\longrightarrow} \frac{\delta_{m}}{\pi} .
$$

Here, $\Omega=-T \ln \mathrm{e}^{-\mathcal{H} / T}$ is the free energy. The phase shift also determines the value of the spectral function at $\omega=T=0$,

$$
A_{m}(0,0)=\frac{\sin ^{2} \delta_{m}}{\pi \Delta} .
$$

The linear susceptibilities $\chi_{m_{1}, m_{2}}$ are also important parameters that determine the Fermi-liquid properties:

$$
\chi_{m_{1}, m_{2}} \equiv \int_{0}^{1 / T} d \tau\left\langle\delta n_{d m_{1}}(\tau) \delta n_{d m_{2}}\right\rangle .
$$

Here, $\delta n_{d m} \equiv n_{d m}-\left\langle n_{d m}\right\rangle$ is the fluctuation of the occupation number, and $\delta n_{d m}(\tau)=\mathrm{e}^{\tau \mathcal{H}} \delta n_{d m} \mathrm{e}^{-\tau \mathcal{H}}$. The derivative of the self-energy with respect to the impurity level also gives the susceptibilities,

$$
\begin{aligned}
\chi_{m_{1}, m_{2}} & =-\frac{\partial^{2} \Omega}{\partial \epsilon_{m_{1}} \partial \epsilon_{m_{2}}}=-\frac{\partial\left\langle n_{d m_{1}}\right\rangle}{\partial \epsilon_{m_{2}}} \\
& \stackrel{T \rightarrow 0}{\longrightarrow} A_{m_{1}}(0,0)\left(\delta_{m_{1}, m_{2}}+\frac{\partial \Sigma_{m_{1}}(0,0)}{\partial \epsilon_{m_{2}}}\right) .
\end{aligned}
$$

The Ward-Takahashi identities relate the linear susceptibilities to the wave function renormalization factor $Z_{m}$ and the vertex function $\Gamma_{m m^{\prime} ; m^{\prime} m}(0,0 ; 0,0)[45,46]$,

$$
\begin{aligned}
& \chi_{m, m}=\frac{A_{m}(0,0)}{Z_{m}}, \frac{1}{Z_{m}} \equiv 1-\left.\frac{\partial \Sigma_{m}(\omega, 0)}{\partial \omega}\right|_{\omega=0}, \\
& \chi_{m, m^{\prime}}=-A_{m}(0,0) A_{m^{\prime}}(0,0) \Gamma_{m m^{\prime} ; m^{\prime} m}(0,0 ; 0,0) .
\end{aligned}
$$

$\Gamma_{m m^{\prime} ; m^{\prime} m}\left(\omega, \omega^{\prime} ; \omega^{\prime}, \omega\right)$ is the $m \neq m^{\prime}$ component of the vertex correction, defined at $T=0$ for the causal Green's functions. Note that the intra-level components for $m=m^{\prime}$ vanish at zero frequencies, $\Gamma_{m m ; m m}(0,0 ; 0,0)=0$, because of the fermionic antisymmetrical properties, i.e., the Pauli exclusion principle.
The renormalized level position $\widetilde{\epsilon}_{m}$ and corresponding resonance width $\widetilde{\Delta}_{m}$ are determined by $Z_{m}$,

$$
\widetilde{\epsilon}_{m} \equiv Z_{m}\left[\epsilon_{m}+\Sigma_{m}(0)\right], \quad \widetilde{\Delta}_{m} \equiv Z_{m} \Delta .
$$

The residual interaction between quasiparticles $\widetilde{U}_{m, m^{\prime}}$ is also an essential Fermi-liquid parameter [47]:

$$
\widetilde{U}_{m, m^{\prime}} \equiv Z_{m} Z_{m^{\prime}} \Gamma_{m m^{\prime} ; m^{\prime} m}(0,0 ; 0,0) \text {. }
$$

The Wilson ratio $R_{m, m^{\prime}}$ corresponds to a dimensionless residual interaction [48], and it generally depends on $m$ and $m^{\prime}$ :

$$
R_{m, m^{\prime}} \equiv 1+\sqrt{\widetilde{A}_{m} \widetilde{A}_{m^{\prime}}} \widetilde{U}_{m, m^{\prime}}
$$

Here, $\widetilde{A}_{m} \equiv A_{m}(0,0) / Z_{m}$ is the density of states of the quasiparticles. Using Eqs. (14)-(17), $R_{m, m^{\prime}}$ can also be expressed in terms of the linear susceptibilities,

$$
R_{m, m^{\prime}}-1=-\frac{\chi_{m, m^{\prime}}}{\sqrt{\chi_{m, m} \chi_{m^{\prime}, m^{\prime}}}} .
$$

we calculate the renormalization factor $Z_{m}$ and residual interaction $\widetilde{U}_{m, m^{\prime}}$ using the NRG, and deduce the Wilson ratio $R_{m, m^{\prime}}$ from Eq. (18). We note that the linear susceptibility $\chi_{m, m}$ determines the $T$-linear specific heat $\mathcal{C}_{\mathrm{dot}}$ due to the impurity,

$$
\mathcal{C}_{\text {dot }}=\gamma T, \quad \gamma=\frac{\pi^{2}}{3} \sum_{m} \chi_{m, m} .
$$

Furthermore, fluctuations of the impurity electron filling are described by the charge susceptibility $\chi_{c} \equiv \sum_{m} \chi_{c, m}$, which can also be expressed in terms of $\widetilde{A}_{m}$ and $\widetilde{U}_{m, m^{\prime}}$ using Eqs. (14) and (15):

$$
\begin{aligned}
\chi_{c, m} & \equiv-\sum_{m^{\prime}} \frac{\partial\left\langle n_{d m}\right\rangle}{\partial \varepsilon_{m^{\prime}}}=\sum_{m^{\prime}} \chi_{m, m^{\prime}} \\
& =\widetilde{A}_{m}(0)\left[1-\sum_{m^{\prime}(\neq m)} \widetilde{U}_{m, m^{\prime}} \widetilde{A}_{m^{\prime}}(0)\right] .
\end{aligned}
$$

The last line shows that the residual interaction $\widetilde{U}_{m, m^{\prime}}$ reduces the free-quasiparticle contributions given by the first term in the right-hand side.

\section{Conductance and nonlinear susceptibilities}

The conductance $g_{\text {tot }}$ through a quantum dot can be expressed in the Landauer form [49-51],

$$
\begin{gathered}
g_{\text {tot }}=\sum_{m=1}^{N} g_{m}, \\
g_{m}=\frac{e^{2}}{h} \frac{4 \Delta_{L} \Delta_{R}}{\Delta_{L}+\Delta_{R}} \int_{-\infty}^{\infty} d \omega\left(-\frac{\partial f(\omega)}{\partial \omega}\right) \pi A_{m}(\omega, T) .
\end{gathered}
$$

Here, $f(\omega)=1 /\left(e^{\omega / T}+1\right)$ is the Fermi distribution function. It has recently been clarified that low-temperature behavior of conductance up to order $T^{2}$ can be determined completely by an extended Fermi-liquid theory [31-33]. This formulation is also applicable to the multilevel Anderson impurity model at arbitrary electron fillings, and the low-temperature expansion 
can be expressed in the following form, for symmetric tunneling couplings $\Delta_{L}=\Delta_{R}[33,34]$,

$$
g_{m}=\frac{e^{2}}{h}\left[\sin ^{2} \delta_{m}+c_{T, m}(\pi T)^{2}+\cdots\right]
$$

Here, the first term in the right-hand side corresponds to the ground-state value that is determined by the transmission probability $\sin ^{2} \delta_{m}$. The coefficient $c_{T, m}$ for the term of order $T^{2}$ consists of two parts [31-34]:

$$
\begin{gathered}
c_{T, m}=\frac{\pi^{2}}{3}\left[w_{T, m}+\theta_{T, m}\right], \\
w_{T, m}=-\cos 2 \delta_{m}\left(\chi_{m, m}^{2}+2 \sum_{m^{\prime}(\neq m)} \chi_{m, m^{\prime}}^{2}\right), \\
\theta_{T, m}=\frac{\sin 2 \delta_{m}}{2 \pi}\left(\chi_{m, m, m}^{[3]}+\sum_{m^{\prime}(\neq m)} \chi_{m, m^{\prime}, m^{\prime}}^{[3]}\right) .
\end{gathered}
$$

Here, $w_{T, m}$ represents the two-body contributions which are determined by the linear susceptibilities $\chi_{m, m^{\prime}}$. The other part $\theta_{T, m}$ represents the three-body contributions, determined by the nonlinear susceptibilities [33,34],

$$
\chi_{m_{1}, m_{2}, m_{3}}^{[3]} \equiv-\int_{0}^{\beta} d \tau_{1} \int_{0}^{\beta} d \tau_{2}\left\langle T_{\tau} \delta n_{d m_{3}}\left(\tau_{3}\right) \delta n_{d m_{2}}\left(\tau_{2}\right) \delta n_{d m_{1}}\right\rangle .
$$

Here, $T_{\tau}$ is the imaginary time ordering operator. This correlation function corresponds also to the derivative of linear susceptibilities:

$$
\chi_{m_{1}, m_{2}, m_{3}}^{[3]}=-\frac{\partial^{3} \Omega}{\partial \epsilon_{m_{1}} \partial \epsilon_{m_{2}} \partial \epsilon_{m_{3}}}=\frac{\partial \chi_{m_{2}, m_{3}}}{\partial \epsilon_{m_{1}}} .
$$

We note that $\chi_{m_{1}, m_{2}, m_{3}}^{[3]}$ vanishes in the case at which the system has both the particle-hole and time-reversal symmetries.

\section{NRG approach to the spectral function and transport coefficients}

The NRG has successfully been applied to multi-orbital quantum dots including CNT dots since a seminal work of Izumida et al. [10,11,37,40,52-55]. Using the NRG, we calculate the renormalized parameters such as the phase shifts of electrons $\delta$, the wave-function renormalization factor $Z$, and the Wilson ratio $R$ [56-58]. The present work uses the NRG to calculate not only the renormalized parameters, but also the linear conductance $g$ and the spectral function $A_{m}(\omega, T)$ [21-23].

The key approximation of the NRG is the logarithmic discretization of the conduction band. A dimensionless parameter $\Lambda(>1)$ controls the discretization. The noninteracting part of the discretized Hamiltonian $\mathcal{H}_{d}^{0}+\mathcal{H}_{T}+$ $\mathcal{H}_{c}$ is transformed into a one-dimensional tight-biding chain with exponentially decaying hopping matrix elements $t_{n} \sim$ $D \Lambda^{-n / 2}$. Then, the total Hamiltonian $\mathcal{H}$ including the interactions can be diagonalized iteratively by adding the states on the tight-biding chain, starting from the impurity site. Owing to the exponential decay of $t_{n}$, high energy states can be discarded at each successive step without affecting low-lying energy states so much. Although this iteration itself is an artificial procedure, it can be interpreted as a process to probe lower energy scale, step by step, stating from high-energy scale [21]. We briefly explain the process of the NRG iteration in Appendix B. Furthermore, we can deduce the quasiparticles parameters $Z_{m}, \widetilde{\epsilon}_{m}$, and $\widetilde{U}_{m, m^{\prime}}$, from asymptotic behaviours of the low-lying eigenvalues near the NRG fixed point (see Appendix C) [22,23,48].

In the present work, we explore the CNT dots in a case where the fourfold degeneracy is not completely lifted by the magnetic field but still a double degeneracy remains for the reason which will be discussed in more detail in Sec. III B.

Our NRG code uses the $\mathrm{U}(1) \otimes[\mathrm{SU}(2) \otimes \mathrm{U}(1)] \otimes \mathrm{U}(1)$ symmetries to explore the $\mathrm{SU}(4)$ to $\mathrm{SU}(2)$ Kondo crossover. The $\mathrm{U}(1) \otimes \mathrm{U}(1) \otimes \mathrm{U}(1) \otimes \mathrm{U}(1)$ symmetries are used to examine how perturbations, i.e., the valley mixing and spin-orbit interaction, affect the crossover because the perturbations break the SU(2) symmetry. The NRG calculations are carried out keeping typically the lowest 4100 states in the truncation procedure, choosing the discretization parameter to be $\Lambda=6.0$. Furthermore, the spectral function and temperaturedependent conductance $[59,60]$ are obtained using the complete Fock-space basis algorithm [24-26], together with the Oliveira $z$ averaging $[59,61]$. For obtaining the spectral functions, we also calculate the higher-order correlation function in order to directly deduce the self-energy $\Sigma_{m}(\omega, T)$ [62]. These supplemental techniques for the dynamical correlations functions are also described in Appendix E.

\section{FIELD-INDUCED SU(4) TO SU(2) CROSSOVER OF KONDO SINGLET STATE}

\section{A. Microscopic description for the CNT-dot levels}

The Hamiltonian $\mathcal{H}$ defined in Eqs. (1)-(4) are described using a representation in which the dot part $\mathcal{H}_{d}^{0}$ has already been diagonalized. However, to see a microscopic background, the other basis set using the spin $(\uparrow, \downarrow)$ and valley $\left(K, K^{\prime}\right)$ degrees of freedom is more suitable.

The valley degrees of freedom capture a magnetic moment along the CNT axis because of the cylindrical geometry of the CNT. This orbital moment couples to an external magnetic field parallel to the CNT axis $[7,28,37,63,64]$. The four levels of the CNT dot are also coupled each other through the spinorbit interaction $\Delta_{\mathrm{SO}}$ and the valley mixing $\Delta_{K K^{\prime}}$. The dot part of the Hamiltonian can be expressed in the following form, using the dot-electron operator $\psi_{d: \ell s}^{\dagger}$ for orbital $\ell\left(=K, K^{\prime}\right)$ and $\operatorname{spin} s(=\uparrow, \downarrow)$,

$$
\mathcal{H}_{d}^{0} \equiv \sum_{\ell, \ell^{\prime}} \sum_{s, s^{\prime}} \psi_{d: \ell s}^{\dagger} H_{d: \ell s, \ell^{\prime} s^{\prime}}^{0} \psi_{d: \ell^{\prime} s^{\prime}}=\boldsymbol{\psi}_{d}^{\dagger} \boldsymbol{H}_{d}^{0} \boldsymbol{\psi}_{d} .
$$

The matrix $\boldsymbol{H}_{d}^{0} \equiv\left\{H_{d: \ell, \ell^{\prime} s^{\prime}}^{0}\right\}$ is given by $[7,28,37]$

$$
\begin{gathered}
\boldsymbol{H}_{d}^{0}=\varepsilon_{d} \mathbf{1}_{\mathrm{s}} \otimes \mathbf{1}_{\mathrm{orb}}+\frac{\Delta_{K K^{\prime}}}{2} \mathbf{1}_{\mathrm{s}} \otimes \boldsymbol{\tau}^{x}+\frac{\Delta_{\mathrm{SO}}}{2} \boldsymbol{\sigma}^{z} \otimes \boldsymbol{\tau}^{z}-\overrightarrow{\mathcal{M}} \cdot \vec{b}, \\
\overrightarrow{\mathcal{M}} \equiv-\frac{1}{2} g_{\mathrm{s}} \overrightarrow{\boldsymbol{\sigma}} \otimes \mathbf{1}_{\mathrm{orb}}-g_{\mathrm{orb}} \mathbf{1}_{\mathrm{s}} \otimes \boldsymbol{\tau}^{z} \vec{e}_{z}
\end{gathered}
$$

Here, $\boldsymbol{\sigma}^{j}=\left\{\sigma_{s s^{\prime}}^{j}\right\}$ and $\boldsymbol{\tau}^{j}=\left\{\tau_{\ell \ell^{\prime}}^{j}\right\}$ for $j=x, y, z$ are the Pauli matrices for the spin and the valley pseudo-spin spaces, respectively. Correspondingly, $\mathbf{1}_{\mathrm{s}}=\left\{\delta_{s s^{\prime}}\right\}$ and $\mathbf{1}_{\mathrm{orb}}=\left\{\delta_{\ell \ell^{\prime}}\right\}$ are the corresponding unit matrices. In a finite magnetic field 
$\vec{b} \equiv \mu_{B} \vec{B}$, where $\mu_{B}$ is the Bohr magneton, both the spin and orbital moments contribute to the magnetization $\overrightarrow{\mathcal{M}}$. The $g$ factor for the spin is $g_{\mathrm{s}}=2.0$. The orbital moment couples to the magnetic field along the nanotube axis, and the orbital Zeeman splitting is given by $\pm g_{\text {orb }}|\vec{b}| \cos \Theta$. Here, $g_{\text {orb }}$ is the orbital Landé factor and $\Theta$ is the angle of the magnetic field relative to the nanotube axis, which is chosen as the $z$ axis with $\vec{e}_{z}$ the unit vector. The one-particle Hamiltonian of the dot levels $\boldsymbol{H}_{d}^{0}$ can be diagonalized via the unitary transform with the matrix $\boldsymbol{U}_{d}=\left(\boldsymbol{u}_{1}, \boldsymbol{u}_{2}, \boldsymbol{u}_{3}, \boldsymbol{u}_{4}\right)$, constructed with the eigenvectors,

$$
\boldsymbol{H}_{d}^{0} \boldsymbol{u}_{m}=\epsilon_{m} \boldsymbol{u}_{m}, \quad \boldsymbol{u}_{m}^{\dagger} \cdot \boldsymbol{u}_{m^{\prime}}=\delta_{m m^{\prime}} .
$$

The operator $d_{m}$ that annihilates an electron in the eigenstate with energy $\epsilon_{m}$ can be expressed in a linear combination of $\psi_{d: \ell s}$ 's via this transform with $\mathcal{U}_{d}$.

For $\Delta_{\mathrm{SO}}=\Delta_{K K^{\prime}}=0$, the spin component of the magnetization becomes parallel to the field $\vec{e}_{\Theta}=\cos \Theta \vec{e}_{z}+\sin \Theta \vec{e}_{x}$ while the orbital component is in the direction along the nanotube axis $(\Theta \leqslant \pi / 2)$. Then, the eigenvalues of $\boldsymbol{H}_{d}^{0}$ can be written as $\epsilon_{1}=\varepsilon_{d}-\left(g_{\text {orb }} \cos \Theta+g_{\mathrm{s}} / 2\right) b, \quad \epsilon_{2}=$ $\varepsilon_{d}-\left(g_{\text {orb }} \cos \Theta-g_{\mathrm{s}} / 2\right) b, \quad \epsilon_{3}=\varepsilon_{d}+\left(g_{\text {orb }} \cos \Theta-g_{\mathrm{s}} / 2\right) b$, and $\epsilon_{4}=\varepsilon_{d}+\left(g_{\text {orb }} \cos \Theta+g_{\mathrm{s}} / 2\right) b$. The eigenstates, for $m=1,2,3$, and 4 , correspond to $\left|K^{\prime} \downarrow_{\vec{b}}\right\rangle,\left|K^{\prime} \uparrow_{\vec{b}}\right\rangle,\left|K \downarrow_{\vec{b}}\right\rangle$, and $\left|K \uparrow_{\vec{b}}\right\rangle$, respectively, with $\uparrow_{\vec{b}}$ and $\downarrow_{\vec{b}}$ the spin defined with respect to the direction along the field $\vec{b}$. Therefore the thermal average of $\overrightarrow{\mathcal{M}}$ can be written in the form

$$
\begin{gathered}
\left\langle\boldsymbol{\psi}_{d}^{\dagger} \overrightarrow{\mathcal{M}} \psi_{d}\right\rangle=\mathcal{M}_{\text {orb }} \vec{e}_{z}+\mathcal{M}_{\mathrm{s}} \vec{e}_{\Theta}, \\
\mathcal{M}_{\text {orb }}=g_{\text {orb }}\left[\left\langle n_{d 1}\right\rangle-\left\langle n_{d 4}\right\rangle+\left\langle n_{d 2}\right\rangle-\left\langle n_{d 3}\right\rangle\right], \\
\mathcal{M}_{\mathrm{s}}=\frac{g_{\mathrm{s}}}{2}\left[\left\langle n_{d 1}\right\rangle-\left\langle n_{d 4}\right\rangle-\left\langle n_{d 2}\right\rangle+\left\langle n_{d 3}\right\rangle\right] .
\end{gathered}
$$

In more general cases, the matrix for $\overrightarrow{\mathcal{M}}$ with respect to one-particle eigenvector $\boldsymbol{u}_{m}$, can be expressed in the following forms using the Feynman theorem:

$$
\vec{K}_{m} \equiv \boldsymbol{u}_{m}^{\dagger} \overrightarrow{\mathcal{M}} \boldsymbol{u}_{m}=-\boldsymbol{u}_{m}^{\dagger} \frac{\partial \boldsymbol{H}_{d}^{0}}{\partial \vec{b}} \boldsymbol{u}_{m}=-\frac{\partial \epsilon_{m}}{\partial \vec{b}} .
$$

The thermal average of the magnetization $\overrightarrow{\mathcal{M}}$ can be written in terms of these matrix elements,

$$
\overrightarrow{\mathcal{M}} \equiv\left\langle\boldsymbol{\psi}_{d}^{\dagger} \overrightarrow{\mathcal{M}} \psi_{d}\right\rangle=\sum_{m} \vec{K}_{m}\left\langle n_{d, m}\right\rangle
$$

Therefore the magnetic susceptibility $\chi_{\mathcal{M}}^{\mu \nu} \equiv \partial \mathcal{M}^{\mu} / \partial b_{v}$, which is not isotropic for nano-tube dots, can be expressed in the following form:

$$
\begin{aligned}
\chi_{\mathcal{M}}^{\mu \nu}= & \sum_{m} \frac{\partial K_{m}^{\mu}}{\partial b_{v}}\left\langle n_{d, m}\right\rangle+\sum_{m, m^{\prime}} K_{m}^{\mu} \frac{\partial \epsilon_{m^{\prime}}}{\partial b_{v}} \frac{\partial\left\langle n_{d, m}\right\rangle}{\partial \epsilon_{m^{\prime}}} \\
= & \sum_{m} \frac{\partial K_{m}^{\mu}}{\partial b_{v}}\left\langle n_{d, m}\right\rangle+\sum_{m, m^{\prime}} K_{m}^{\mu} K_{m^{\prime}}^{v} \chi_{m, m^{\prime}}, \\
= & \sum_{m} \frac{\partial K_{m}^{\mu}}{\partial b_{v}}\left\langle n_{d, m}\right\rangle+\sum_{m} K_{m}^{\mu} K_{m}^{v} \widetilde{A}_{m} \\
& -\sum_{m \neq m^{\prime}} K_{m}^{\mu} K_{m^{\prime}}^{v} \widetilde{A}_{m} \widetilde{A}_{m^{\prime}} \widetilde{U}_{m m^{\prime}} .
\end{aligned}
$$

Here, the last term in the right-hand side represents the contributions of the residual interaction, or the vertex corrections. Since the Wilson ratio $R_{m, m^{\prime}}$ given in Eq. (18) depends on the residual interaction, $R_{m, m^{\prime}}$ relates to the magnetic susceptibilities.

\section{B. CNT level structure and field-induced crossover}

In recent experiments reported in Refs. [19,35], nonlinear current and current noise were measured for a CNT dot with the orbital Landé factor $g_{\text {orb }} \approx 4$ at finite magnetic fields with an angle $\Theta \approx 75^{\circ}$. These values of $g_{\text {orb }}$ and $\Theta$ imply that the magnitude of the orbital Zeeman splitting becomes almost the same as the spin Zeeman splitting in this particular situation,

$$
g_{\text {orb }} \cos \Theta \approx \frac{1}{2} g_{\text {orb }}=1 .
$$

This situation can take place for CNT dots as the orbital Landé factor $g_{\text {orb }}$ depends significantly on the diameter of nanotube and takes a value around $g_{\text {orb }} \sim 10$ [7]. In the case where this matching of the orbital and spin Zeeman splittings is satisfied, the energy level of the dot has a double degeneracy which remains unlifted in magnetic fields:

$$
\epsilon_{1}=\varepsilon_{d}-2 b, \quad \epsilon_{2} \equiv \epsilon_{3}=\varepsilon_{d}, \quad \epsilon_{4}=\varepsilon_{d}+2 b .
$$

In this case, the occupation numbers of the degeneracy become the same $\left\langle n_{d 2}\right\rangle=\left\langle n_{d 3}\right\rangle$. Thus both the orbital and spin magnetizations are determined by the occupation numbers of the other two levels $m=1$ and $m=4: \mathcal{M}_{\text {orb }}=g_{\text {orb }} \mathcal{M}_{14}$ and $\mathcal{M}_{\mathrm{s}}=\frac{g_{\mathrm{s}}}{2} \mathcal{M}_{14}$, with

$$
\mathcal{M}_{14} \equiv\left\langle n_{d 1}\right\rangle-\left\langle n_{d 4}\right\rangle .
$$

We note that $\Delta_{\text {So }}$ and $\Delta_{K K^{\prime}}$ are less important for the examined CNT dot $[19,35,36]$. In this situation, the system has an $\mathrm{SU}(2)$ rotational symmetry defined with respect to the degenerate states in the middle, and the U(1) symmetry that conserves the sum of the occupation numbers $n_{2}+n_{3}$, in addition to the other two $\mathrm{U}(1)$ symmetries corresponding to $n_{1}$ and $n_{4}$ for the levels $m=1$ and $m=4$, respectively. Therefore the SU(4) symmetry that the total Hamiltonian has at zero field breaks down to the $\mathrm{U}(1)_{m=1} \otimes$ $[\mathrm{SU}(2) \otimes \mathrm{U}(1)]_{m=2,3} \otimes \mathrm{U}(1)_{m=4}$ symmetry at finite magnetic fields. This $\mathrm{SU}(2)$ symmetric part plays a central role in the field-induced $\mathrm{SU}(4)$ to $\mathrm{SU}(2)$ Kondo crossover, occurring at half-filling point $N_{d}=2$. At this point, due to the matching condition given in Eq. (41), the Hamiltonian $\mathcal{H}$ is invariant under an extended electron-hole transformation described by Eq. (A5).

\section{Comparison of NRG and experiments results: gate-voltage dependence at finite $B$ or $T$}

We have shown in the previous work $[35,36]$ that the level scheme, given in Eq. (41), nicely explains the field-induced $\mathrm{SU}(4)$ to SU(2) Kondo crossover observed at half-filling $N_{d}=$ 2 where two electrons occupy the local levels of the CNT dot. The Coulomb interaction for this CNT dot is estimated to be $U \approx 6 \mathrm{meV}$, and the hybridization energy is $\Delta \equiv \Delta_{L}+\Delta_{R} \approx$ $0.9 \mathrm{meV}$. Its asymmetric factor is $4 \Delta_{L} \Delta_{R} /\left(\Delta_{L}+\Delta_{R}\right)^{2}=$ 0.92 . The energy scales the interaction as $U /(\pi \Delta)=2.0$. The 
SU(4) Kondo temperature for the scaled $U$ is $T_{K}^{\mathrm{SU}(4)} / \Delta=$ 0.41 which corresponds to $T_{K}=4.3 \mathrm{~K}$ in a real scale. These values of $U$ and $\Delta$ are much larger than the value of the vally mixing $\Delta_{K K^{\prime}}$ and than that of the spin-orbit interaction $\Delta_{\text {So }}$. The experimental values of $\Delta_{K K^{\prime}}$ and $\Delta_{\mathrm{SO}}$ are $\Delta_{K K^{\prime}} \simeq \Delta_{\mathrm{SO}} \approx$ $0.2 \mathrm{meV}$. We note that these values depend on the detailed structures of carbon nanotube samples. Furthermore, it has also estimated in the experiment that the orbital Zeemann coupling as $g_{\text {orb }} \cos \theta \simeq 0.7$ [35], which is not so far from the matching condition $g_{\text {orb }} \cos \theta=1$, described in Eq. (40).

We fist of all discuss the magnetoconductance for the case $g_{\text {orb }} \cos \theta=1$ and $\Delta_{K K^{\prime}}=\Delta_{\text {SO }}=0$, at which an ideal SU(4) to $\mathrm{SU}(2)$ Kondo crossover occurs. Figure 1(a) compares the NRG results of the conductance at $T=0$ with the experimental results obtained at $T=16 \mathrm{mK}$. The values of $U$ and $\Delta$ for the NRG calculations are the experimental values. The comparisons which have been done also in Ref. [35] show that the NRG results nicely agree with the experimental results. We can clearly see in this figure that the Kondo ridge emerges near half-filling $V_{\mathrm{g}} \simeq 26 \mathrm{~V}$ in the absence and presence of magnetic fields. Its height reduces from the SU(4) value $3.68 \mathrm{e}^{2} / h$ to the $\mathrm{SU}(2)$ value $1.84 \mathrm{e}^{2} / h$ as magnetic field increases. This reduction of the height implies that the observed SU(2) behavior is caused by the doubly degenerate states, which are labeled as $m=2$ and 3 in Eq. (41), are shifted towards the Fermi level in the half-filled case where $\epsilon_{d}=-3 U / 2$. Furthermore, the additional subpeaks, which emerge outside the Kondo ridge for large magnetic fields $B \gtrsim 4 \mathrm{~T}$, can also be regarded as the resonances corresponding to the other two nondegenerate levels, labeled as $m=1$ and 4 . Note that the Kodo ridges corresponding to the $1 / 4$ and $3 / 4$ fillings are not so pronounced at $B=0$ because the Coulomb interaction for this CNT $\operatorname{dot} U /(\pi \Delta)=2.0$ is not very large.

We also examine the case where the matching condition described in Eq. (40) cannot be satisfied, taking the parameters such that $\Delta_{K K^{\prime}}=\Delta_{\mathrm{SO}}=0.2 \mathrm{meV}$ and $g_{\text {orb }} \cos \theta=0.7{ }^{1}$ At zero field $b=0$, the level structure is given by

$$
\begin{aligned}
& \epsilon_{1}=\epsilon_{2}=\epsilon_{d}-\frac{1}{2} \sqrt{\Delta_{K K^{\prime}}^{2}+\Delta_{\mathrm{SO}}^{2}}, \\
& \epsilon_{3}=\epsilon_{4}=\epsilon_{d}+\frac{1}{2} \sqrt{\Delta_{K K^{\prime}}^{2}+\Delta_{\mathrm{SO}}^{2}} .
\end{aligned}
$$

The value of the gap due to the spin-orbit interaction and valley mixing is $\sqrt{\Delta_{K K^{\prime}}^{2}+\Delta_{\text {SO }}^{2}} \simeq 0.28 \mathrm{meV}$, which is smaller than the resonance width. Figure 1(b) shows the NRG results, obtained taking the other parameters the same as those for Fig. 1(a), i.e., $U /(\pi \Delta)=2.0, \Delta=0.9 \mathrm{meV}$. The broad peak emerges also in Fig. 1(b) although its peak value $3.14 \mathrm{e}^{2} / h$ is smaller than that the value $3.68 \mathrm{e}^{2} / h$, which is for the case of $\Delta_{K K^{\prime}}=\Delta_{\mathrm{SO}}=0$ and $g_{\text {orb }} \cos \theta=1$. The flat structure is still preserved in small magnetic fields up to $B \approx 5 T$. This preservation shows that the $\mathrm{SU}(4)$ to $\mathrm{SU}(2)$ Kondo crossover is robust against the perturbations. The larger magnetic fields $B \gtrsim 5 T$ split the peak into two peaks. Furthermore, other two sub peaks grow with increasing $B$, and thus the four distinct peaks emerge. Figure 1(b) shows that the magnetoconductance for $B=10 \mathrm{~T}$ has the four peaks.

\footnotetext{
${ }^{1}$ See Supplemental Material of Ref. [35].
}
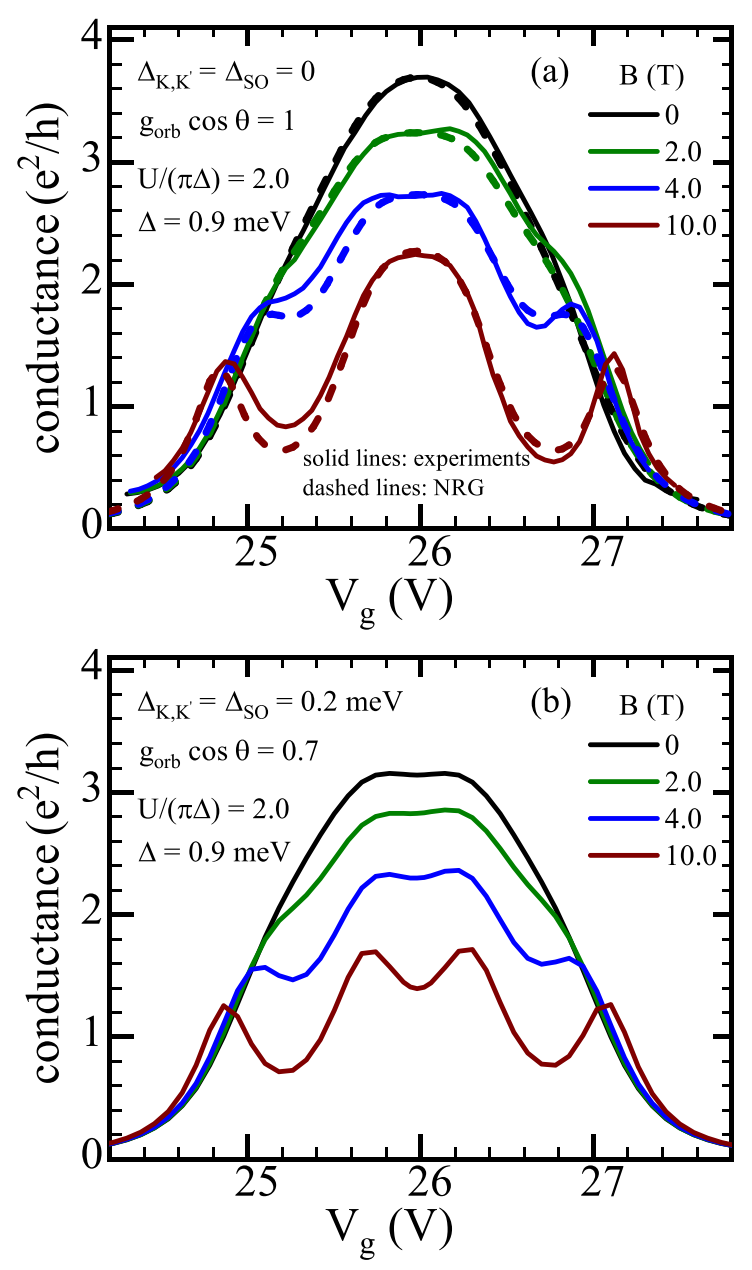

FIG. 1. (a) and (b) plot the zero temperature conductance as functions of experimental gate voltages $V_{\mathrm{g}}$ for four values of magnetic fields: $B=0,2,4$, and $10 \mathrm{~T}$. In the CNT dot where the experiments observed the crossover, the experimental $V_{\mathrm{g}}$ relates to theoretical the theoretical $\epsilon_{d}$ via a linear relation: $V_{\mathrm{g}}=$ $\left(0.8 \epsilon_{d} / U+27.18\right)$ in units of volt. The asymmetric factor in Eq. (23) and the Coulomb interaction are respectively $4 \Delta_{L} \Delta_{R} / \Delta^{2}=$ 0.92 and $U /(\pi \Delta)=2.0$ for each two figure. Here, $\Delta \equiv \Delta_{L}+\Delta_{R}=$ $0.9 \mathrm{meV}$. In (a), the dashed lines and solid lines respectively represent NRG and experimental results. The NRG results plotted in (a) are for parameters: $\Delta_{\mathrm{SO}}=\Delta_{K, K^{\prime}}=0, g_{\text {orb }} \cos \theta=1$. The experimental results have been obtained at $T=16 \mathrm{mK}[19,35]$, which is much lower than $T_{K}^{\mathrm{SU}(4)}=4.3 \mathrm{~K}$, the Kondo temperature for the half-filled case (at $V_{\mathrm{g}} \simeq 26 \mathrm{mV}$ ). (b) plots only NRG results by solid lines. The parameters for the results are $\Delta_{\mathrm{SO}}=\Delta_{K, K^{\prime}}=0.2 \mathrm{meV}$ and $g_{\text {orb }} \cos \theta=0.7$.

Our NRG results for the CNT dots, reported so far, were restricted to ground-state properties. The present work sheds light also on the finite-temperature and dynamic properties of the Kondo crossover. Figure 2 compares the experimental results [35] of the zero-field conductance measured at $T=$ $16 \mathrm{mK}, 2 \mathrm{~K}$, and $4.5 \mathrm{~K}$ with the corresponding NRG results, calculated for slightly lower temperatures $T=0 \mathrm{~K}, 1.7 \mathrm{~K}$, and $3.8 \mathrm{~K}$ to demonstrate how these comparisons work at the best. We see a reasonable agreement between the theoretical results and experimental results. The height of the Kondo ridge emerging near half-filling $V_{g} \simeq 26 V$ decreases as temperature 


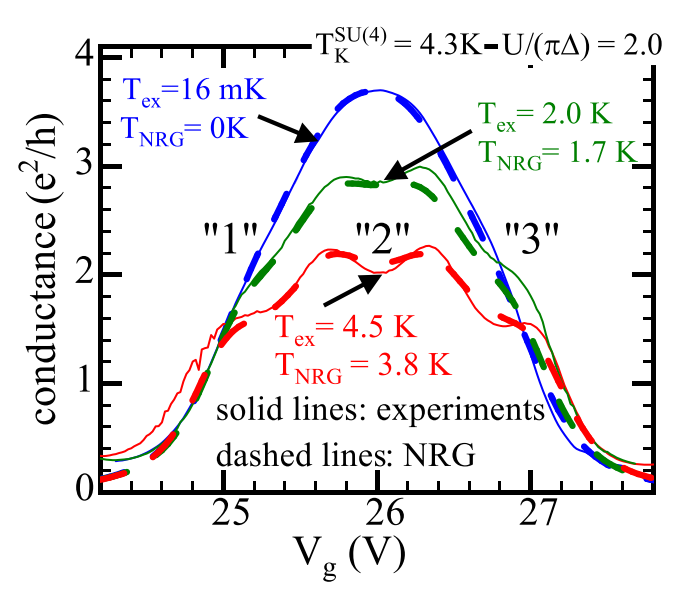

FIG. 2. Zero-field, $B=0$, conductance at finite temperatures: experimental (solid line) and NRG (dashed line) results are plotted vs $V_{\mathrm{g}}$. The experimental data has been obtained at $T=16 \mathrm{mK}$, $2 \mathrm{~K}$, and $4.5 \mathrm{~K}$ [19]. For NRG calculations, slightly lower temperatures $T=0,1.7$, and $3.8 \mathrm{~K}$ are chosen. The other parameters are the same as those used for Fig. 1. The numbers "3," " 2 ," and "1" shown in these two figures represent the electron filling $N_{d}$ at corresponding $V_{g}$.

increases. At temperatures of order $T \sim T_{K}^{\mathrm{SU}(4)}=4.3 \mathrm{~K}$, Four peaks corresponding to the Coulomb oscillation emerge. This agreement also indicates that the theory of the SU(4) Kondo effect can explain the experimental results of the conductance.

\section{Scaling behavior of SU(4) conductance at quarter and half-filling}

In this section, we examine scaling behavior of the SU(4) conductance as functions of temperature especially at quarterfilling $N_{d}=1$ and half-filling $N_{d}=2$. In Fig. 2 , the valley is quarter and half-filled at gate voltages, $V_{g} \simeq 25 \mathrm{~V}$ and $V_{g} \simeq$ $26 V$, respectively. The first value of $V_{g}$ corresponds to the theoretical value $\epsilon_{d} / U=-1 / 2$, and the second one corresponds to $\epsilon_{d} / U=-3 / 2$.

Since the $T^{2}$ coefficient $c_{T, m}$ of conductance given in Eq. (25) depends on linear and nonlinear susceptibilities, we discuss properties of these susceptibilities to examine the scaling. In the $\mathrm{SU}(N)$ symmetric case at which the $N$ impurity levels are degenerate $\epsilon_{m} \equiv \epsilon_{d}$, the linear susceptibilities have only two independent elements: a diagonal element $\chi_{m, m}$ and the off-diagonal element $\chi_{m, m^{\prime}}$ for $m \neq m^{\prime}$. The diagonal element determines a characteristic energy scale $T^{*}$,

$$
T^{*} \equiv \frac{1}{4 \chi_{m, m}} .
$$

In the particle-hole symmetric case $\epsilon_{d} / U \rightarrow-(N-1) / 2$, it corresponds the renormalized width of the Kondo resonance as $T^{*} \rightarrow(\pi / 4) \widetilde{\Delta}$. At any arbitrary electron fillings, $T^{*}$ scales the impurity specific heat defined in Eq. (20) as $\mathcal{C}_{\text {dot }}=\frac{N \pi^{2}}{12}\left(T / T^{*}\right)$.

In the $\mathrm{SU}(N)$ symmetric case, the Wilson ratio $R$ defined in Eq. (18) also takes a simplified form $R-1=-\chi_{m, m^{\prime}} / \chi_{m, m}$, where the flavour index has been dropped in the left-hand side. The Wilson ratio takes the maximum value in the strong coupling limit $U \rightarrow \infty$ at integer filling points:

$$
R_{\mathrm{SU}(N)}^{\max }-1 \stackrel{U \rightarrow \infty, N_{d} \rightarrow \text { integer }}{\longrightarrow} \frac{1}{N-1} .
$$

This is because the charge fluctuations are suppressed in this limit, and the charge susceptibility defined in Eq. (21) vanishes: $\chi_{c, m}=\chi_{m, m}+(N-1) \chi_{m, m^{\prime}} \rightarrow 0$.

The nonlinear susceptibilities have three independent elements in the $\mathrm{SU}(N)$ symmetric case for $N>2: \chi_{m, m, m}^{[3]}$, $\chi_{m, m^{\prime}, m^{\prime}}^{[3]}$, and $\chi_{m, m^{\prime}, m^{\prime \prime}}^{[3]}$, where $m \neq m^{\prime}, m^{\prime} \neq m^{\prime \prime}$, and $m \neq m^{\prime \prime}$. We note that the derivative of the diagonal linear susceptibility $\chi_{m, m}$ with respect to $\epsilon_{d}$ can be related to the first two elements, as

$$
\frac{\partial \chi_{m, m}}{\partial \epsilon_{d}}=\chi_{m, m, m}^{[3]}+(N-1) \chi_{m, m^{\prime}, m^{\prime}}^{[3]}
$$

We next revisit the temperature dependence of conductance for the $\operatorname{SU}(N)$ Anderson model, which has been previously studied in detail by Anders et al. [12], taking in a recent Fermiliquid viewpoint [31-34]. The low-energy expansion, given in Eqs. (24) and (25), takes the following form in the $\mathrm{SU}(N)$ symmetric case,

$$
\begin{gathered}
g_{\text {tot }}=\frac{N e^{2}}{h}\left[\sin ^{2} \delta-C_{T}\left(\frac{\pi T}{T^{*}}\right)^{2}+\cdots\right], \\
C_{T} \equiv \frac{\pi^{2}}{48}\left(W_{T}+\Theta_{T}\right) .
\end{gathered}
$$

The two-body contributions $W_{T}$ and three-body contributions $\Theta_{T}$, defined respectively in Eqs. (26) and (27), can be rewritten in the following form using also Eq. (47),

$$
\begin{gathered}
W_{T} \equiv-\left[1+2(N-1)(R-1)^{2}\right] \cos 2 \delta, \\
\Theta_{T}=\frac{\sin 2 \delta}{2 \pi} \frac{1}{\chi_{m, m}^{2}} \frac{\partial \chi_{m, m}}{\partial \epsilon_{d}} .
\end{gathered}
$$

Figures 3(a) and 3(b) show the temperature dependence of $g_{\text {tot }}$ at half-filling and quarter-filling, respectively. We choose four values of the interaction, $U /(\pi \Delta)=2.0,3.0,4.0$, and 5.0. The first value is the experimental value for the valley where the SU(4) Kondo effect occurs. In other valleys, the experimental values of $U /(\pi \Delta)$ can be larger than 2.0 , and we also consider the larger interaction cases. The temperatures are scaled by the Kondo energy scales $T^{*}$ defined in Eq. (45) for each $N_{d}$ and $U$. In each of the two figures, we find that the scaled conductance curves collapse into a single curve over a wide range of temperatures $T \lesssim T^{*}$ for $U /(\pi \Delta) \gtrsim 3.0$, and thus the conductance shows the universality for each filling.

To clarify the filling dependence of the universality, we replot the curves of quarter and half filling in Fig. 3(c). For the two curves, we choose the largest $U$ among the four, $U /(\pi \Delta)=5.0$. We find that whereas these two curves almost overlap each other around $T \simeq T^{*}$, the conductance of quarter-filling is slightly larger than that of half-filling at low-temperatures $T<T^{*}$, especially around $T \simeq 0.1 T^{*}$. The inset of Fig. 3(c) clearly shows the different behavior depending on the filling.

This filling dependence of the scaling can be explained by the Fermi-liquid theory [31-34]. We have recently clarified using also the NRG that the derivative of the diagonal 

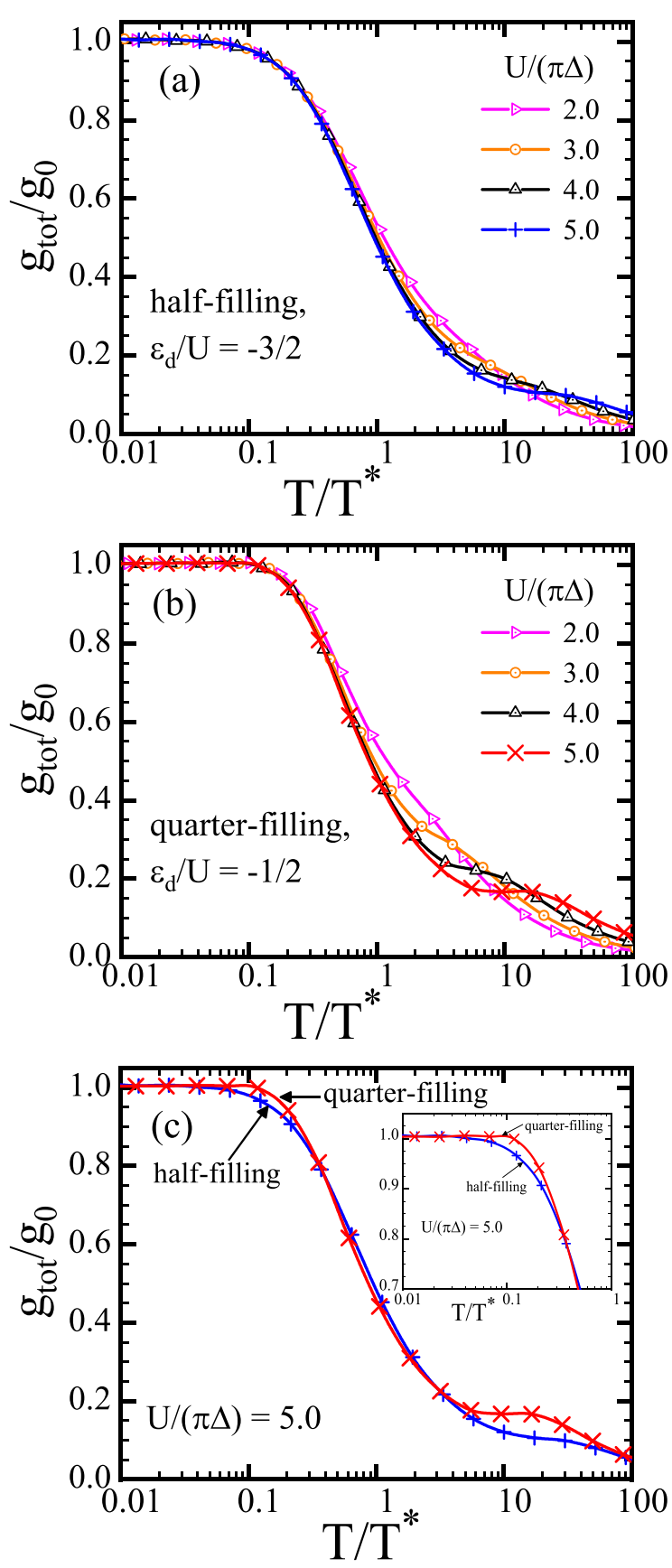

FIG. 3. SU(4) conductance curves are plotted as functions of temperature $T$. (a) and (b) show the results at half-filling and quarterfilling, respectively. In these figures, the conductance curves are plotted for four values of the interaction, $U /(\pi \Delta)=2.0,3.0,4.0$, and 5.0. (c) shows the curves of half-filling and quarter-filling for the largest value $U /(\pi \Delta)=5.0$. The inset of (c) is an enlarged view for $0.01 \leqslant T / T^{*} \leqslant 1$. This inset clearly shows that the $T^{2}$ coefficient $C_{T}$ given in Eq. (49) vanishes at quarter-filling whereas that at half-filling does not. In (a)-(c), the $x$ axis is scaled by the Kondo temperature $T^{*} \equiv 1 /\left(4 \chi_{m, m}\right)$. The values of $T^{*} / \Delta$ at halffilling are $0.41,0.29,0.20$, and 0.13 for $U /(\pi \Delta)=2.0,3.0,4.0$, and 5.0, respectively. Similarly, the values at quarter-filling are $0.82,0.57,0.37$, and 0.23 . The $\mathrm{y}$-axis is normalized by the zero temperature values of conductance, $g_{0}=\left(4 e^{2} / h\right) \sin ^{2} \delta$. At halffilling, $\sin ^{2} \delta \equiv 1$ for any value of $U$, and at quarter-filling, $\sin ^{2} \delta=$ $0.56,0.55,0.54$, and 0.54 . susceptibility $\left|\partial \chi_{m, m} / \partial \epsilon_{d}\right|$ becomes much smaller than $\chi_{m, m}^{2}$ in a wide region of electron fillings $1 \lesssim N_{d} \lesssim 3$ for large interactions $U \gg \Delta$ [34]. From this result, it follows that the three-body contributions $\Theta_{T}$ given by Eq. (51) almost vanish in the same region, and the $T^{2}$ conductance in this case is determined by the two-body contributions, as

$$
C_{T} \simeq-\left(\pi^{2} / 48\right)\left[1+6(R-1)^{2}\right] \cos \left(\pi N_{d} / 2\right) .
$$

Thus $C_{T}$ becomes 0 at quarter filling $N_{d}=1$, whereas it is finite $C_{T}=\left(\pi^{2} / 48\right)\left[1+6(R-1)^{2}\right]$ at half-filling $N_{d}=$ 2. Therefore the conductance at $N_{d}=1$ persists the zero temperature value at $0 \leqslant T / T^{*} \simeq 0.1$ as shown in the inset of Fig. 3(c). At $N_{d}=2$, the coefficient approaches $C_{T}^{\infty}=$ $5 \pi^{2} / 144 \simeq 0.3427$ at $U \rightarrow \infty$. This is because the Wilson ratio for $N=4$ saturates to the strong coupling limit value $R_{\mathrm{SU}(4)}^{\max }-1 \rightarrow 1 / 3$. Already at $U /(\pi \Delta)=3.0$, the coefficient is given by $C_{T} \simeq 0.34$, which is very close to the strongcoupling value $C_{T}^{\infty}$.

\section{EVOLUTION OF QUASIPARTICLES ALONG THE FIELD-INDUCED CROSSOVER}

The Fermi-liquid parameters for renormalized quasiparticles describe low energy properties of quantum dots. The phase shift $\delta_{m}$, which is the primary parameter, corresponds to zero temperature transmission probability. The renormalization factor $Z_{m}$ and the Wilson ratio $R_{m, m^{\prime}}$ are also important parameters to examine the higher-order properties of the Fermi-liquid states. We describe how these and related parameters evolve along a field induced crossover from the SU(4) to SU(2) Kondo singlet state. The crossover occurs for the dot levels defined in Eq. (41). Specifically, we consider the half-filled case corresponding to the point $V_{\mathrm{g}} \simeq 26 \mathrm{~V}$ in the middle of the Kondo ridge seen in Fig. 1. The center of the dot levels is chosen to be $\varepsilon_{d}=-(3 / 2) U$, and thus the average number of electrons in the dot levels conserves in a way such that $\left\langle n_{d 2}\right\rangle=\left\langle n_{d 3}\right\rangle=1 / 2$ and $\left\langle n_{d 1}\right\rangle+\left\langle n_{d 4}\right\rangle=1$ at finite magnetic fields.

We examine two different values for the Coulomb interaction in the following: (i) $U /(\pi \Delta)=2.0$ and (ii) $U /(\pi \Delta)=$ 4.0. The first one, (i), simulates the situation of the CNT dot, in which the field-induced crossover has been observed and the parameters have been estimated as $U \approx 6 \mathrm{meV}$ and $\Delta \approx$ $0.9 \mathrm{meV}[19,35]$. We can see more clearly the renormalization effects due to strong correlations in the second case (ii).

\section{A. Fermi-liquid parameters for the real CNT dot}

First of all, we consider the case $U /(\pi \Delta)=2.0$ that is estimated by the recent experiments. The NRG results for this case are shown in Fig. 4. Figure 4(a) shows the transmission probability $\mathcal{T}_{m}(0)=\sin ^{2} \delta_{m}$ and magnetization $\mathcal{M}_{14} \equiv$ $\left\langle n_{d 1}\right\rangle-\left\langle n_{d 4}\right\rangle$, as a function of magnetic field $b$ at half-filling. The degenerate levels, $m=2$ and 3 , keep their positions just on the Fermi level for finite magnetic fields, and show the unitary limit transport $\sin ^{2} \delta_{m}=1$ as $\delta_{2}=\delta_{3}=\pi / 2$. The magnetic field partly lifts the degeneracy and the other two states, $m=1$ and 4 . For these orbitals, $\sin ^{2} \delta_{m}$ decreases as magnetic field increases. The magnetization $\mathcal{M}_{14}$, which in the present case is determined by the occupation number or 

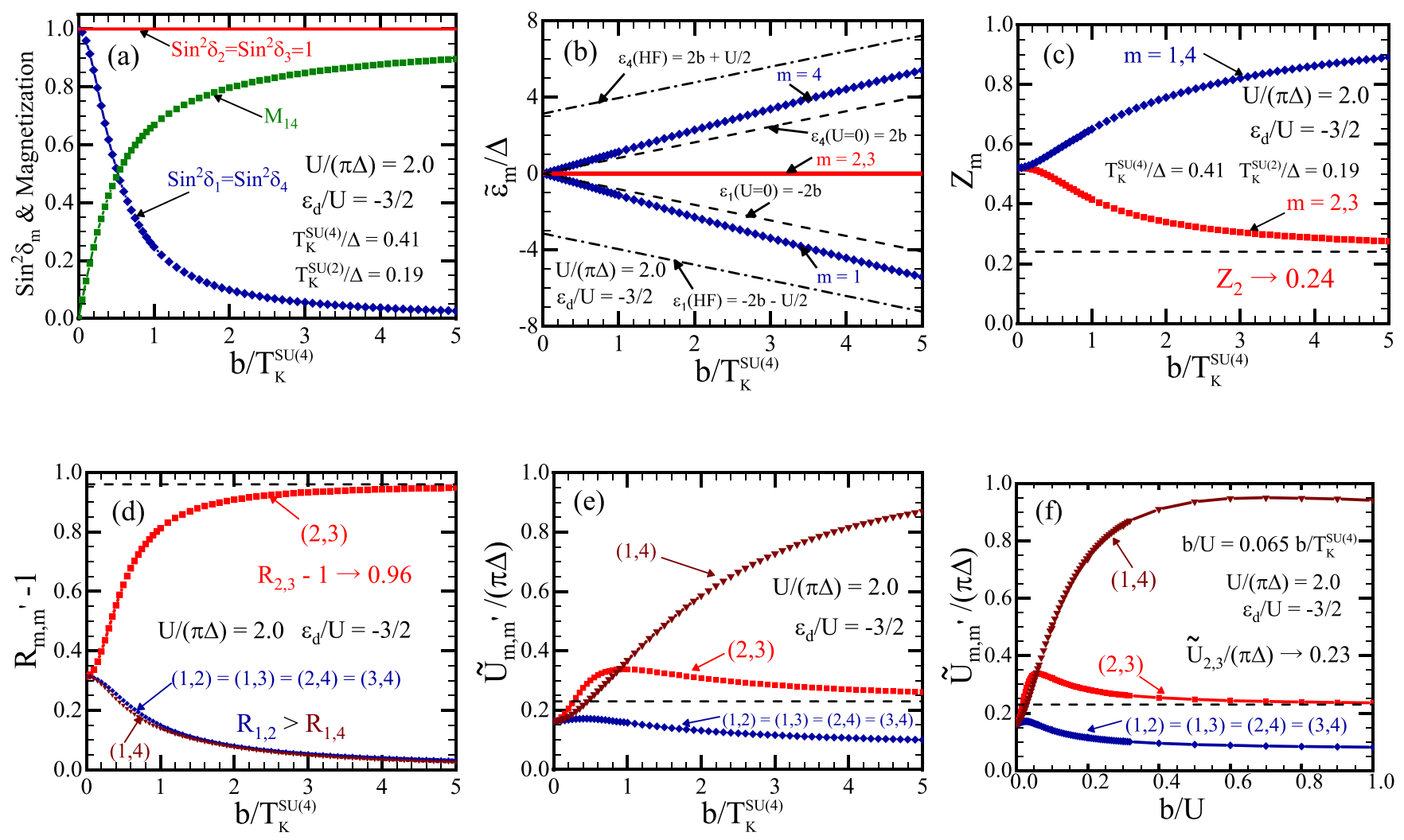

FIG. 4. (a) $\sin ^{2} \delta_{m}$ and magnetization $\mathcal{M}_{14}=\left\langle n_{d 1}\right\rangle-\left\langle n_{d 4}\right\rangle$, (b) renormalized level position $\widetilde{\varepsilon}_{m}$, (c) renormalization factor $Z_{m}$, (d) Wilson ratio $R_{m, m^{\prime}}-1$, and (e) residual interaction $\widetilde{U}_{m, m^{\prime}}$ are plotted as functions of magnetic field $b$ at half-filling $\varepsilon_{d} / U=-3 / 2$ for $U /(\pi \Delta)=2.0$. the $x$ axis in (a)-(e) is scaled by the SU(4) Kondo temperature $T_{K}^{\mathrm{SU}(4)}=0.41 \Delta=(0.065 U)$ determined at $b=0$. In (f) the axis is scaled by $U$ for examining the behavior of $\widetilde{U}_{m, m^{\prime}}$ at larger magnetic fields $b>T_{K}^{\mathrm{SU}(4)}$. The dot levels $\epsilon_{m}$ are chosen in a such way that is described in Eq. (41). In (b), the dashed lines indicate the bare Zeemann splitting, and the dash-dotted lines indicate the mean-field splitting $\varepsilon_{1}^{\mathrm{HF}}=-(2 b+U / 2)$ and $\varepsilon_{4}^{\mathrm{HF}}=(2 b+U / 2)$. In a similar way, the dashed lines in (c)-(f) indicate the $\mathrm{SU}(2)$ symmetric values of $Z_{2} \rightarrow 0.23, R_{2,3} \rightarrow 1.96$, and $\widetilde{U}_{2,3} / \pi \Delta \rightarrow 0.026$, respectively.

these two levels, increases as the magnetic field increases. It saturates to $\mathcal{M}_{14} \rightarrow 1$ in the limit of $b \rightarrow \infty$, and the charge fluctuations are suprressed as $\left\langle n_{d, 1}\right\rangle \rightarrow 1$ and $\left\langle n_{d, 4}\right\rangle \rightarrow 0$.

Figure 4(b) shows the renormalized resonance level position $\widetilde{\varepsilon}_{m}$ as a function of magnetic field $b$. The twofold degenerate states at the center, $\widetilde{\varepsilon}_{2}=\widetilde{\varepsilon}_{3}=0$, remain just on the Fermi level at arbitrary magnetic fields. The other two levels, $\widetilde{\varepsilon}_{1}$ and $\widetilde{\varepsilon}_{4}$ move away from the Fermi level as $b$ increases. Slopes of them are steeper than those for the noninteracting electrons $2 b$ (dashed line). In the large field limit $b \rightarrow \infty$, the renormalized level positions approach the one described in the mean-field theory, i.e., $\varepsilon_{1}^{\mathrm{HF}}=-(2 b+U / 2)$ and $\varepsilon_{4}^{\mathrm{HF}}=$ $(2 b+U / 2)$. These asymptotic form can be obtained as follows, substituting the mean values $\left\langle n_{d 1}\right\rangle=1,\left\langle n_{d 4}\right\rangle=0$ and $\left\langle n_{d 2}\right\rangle=\left\langle n_{d 3}\right\rangle=1 / 2$ into the dot-part of the Hamiltonian with $\varepsilon_{d}=-(3 / 2) U$

$$
\begin{aligned}
\mathcal{H}_{d}^{0}+\mathcal{H}_{U}= & 2 b\left(n_{d 4}-n_{d 1}\right)-\frac{3 U}{2}\left(n_{d 2}+n_{d 3}+n_{d 1}+n_{d 4}\right) \\
& +U\left[n_{d 2} n_{d 3}+n_{d 1} n_{d 4}+\left(n_{d 2}+n_{d 3}\right)\left(n_{d 1}+n_{d 4}\right)\right] \\
& \stackrel{b \rightarrow \infty}{\longrightarrow} U\left[n_{d 2} n_{d 3}-\frac{1}{2}\left(n_{d 2}+n_{d 3}\right)\right] \\
& +\left(2 b+\frac{U}{2}\right)\left(n_{d 4}-n_{d 1}\right)+\text { const. }
\end{aligned}
$$

Here, the Coulomb interaction between the orbitals $m=2$ and 3 is kept undecoupled. This asymptotic Hamiltonian also shows that the symmetric SU(2) Anderson model describes the Fermi-liquid properties of these two orbitals.

The magnetic field dependence of the wavefunction renormalization factors $Z_{m}$ plotted in Fig. 4(c) more clearly shows the crossover. At finite magnetic fields, only two of the four $Z_{m}$ 's become independent: $Z_{2}=Z_{3}$ and $Z_{1}=Z_{4}$ because of the particle-hole symmetry given in Eq. (A5). The first one is for the degenerate levels remaining on the Fermi level, and the second one is for the levels moving away from the Fermi level. At zero field, where the system has the SU(4) symmetry, these two factors for the different orbitals become identical each other: $Z_{2}=Z_{1}=Z_{\mathrm{SU}(4)}=0.52$ for $U /(\pi \Delta)=2.0$. Substituting this $\mathrm{SU}(4)$ value into Eq. (45) gives the $\mathrm{SU}(4)$ Kondo energy scale $T_{K}^{\mathrm{SU}(4)} / \Delta=0.41$. Many-body effects significantly renormalize $Z_{2}$ from the $\mathrm{SU}(4)$ value as magnetic field increases. In the limit of $b \rightarrow \infty$, it approaches the $\mathrm{SU}(2)$ symmetric value $Z_{\mathrm{SU}(2)}=0.23$, which determines the $\mathrm{SU}(2)$ Kondo energy scale $T_{K}^{\mathrm{SU}(2)} / \Delta=0.19$. The many-body effects become less important for $Z_{1}$ with increasing field, and $Z_{1}$ approaches the noninteracting value $Z_{1} \rightarrow 1$ for the large magnetic field. 
In order to clarify the many-body effects between electrons occupying the different orbitals, we also examine the orbital dependent Wilson ratio $R_{m, m^{\prime}}$ and corresponding residual interaction $\widetilde{U}_{m, m^{\prime}}$. Figures $4(\mathrm{~d})$ and $4(\mathrm{e})$ respectively show $R_{m, m^{\prime}}-1$ and $\widetilde{U}_{m, m^{\prime}}$ as functions of $b / T_{K}^{\mathrm{SU}(4)}$. Magnetic field dependences of $\widetilde{U}_{m, m^{\prime}}$ are plotted also in Fig. 4(f), where the magnetic field is scaled by $U$ to examine behaviours of $\widetilde{U}_{m, m^{\prime}}$ at larger fields $b \gg T_{K}^{\mathrm{SU}(4)}$. Owing to the particle-hole symmetry, only three of the six $\widetilde{U}_{m, m^{\prime}}$ are independent: $\widetilde{U}_{2,3}$, $\widetilde{U}_{1,4}$, and $\widetilde{U}_{1,2}=\widetilde{U}_{1,3}=\widetilde{U}_{2,4}=\widetilde{U}_{3,4}^{m, m^{\prime}}$. Correspondingly, three independent parameters of the Wilson ratios, $R_{2,3}, R_{1,4}$, and $R_{1,2}$, can be deduced from Eq. (18):

$$
\begin{gathered}
R_{2,3}-1=\frac{1}{Z_{2}} \frac{\widetilde{U}_{2,3}}{\pi \Delta}, \\
R_{1,4}-1=\frac{\sin ^{2} \delta_{1}}{Z_{1}} \frac{\widetilde{U}_{1,4}}{\pi \Delta}, \\
R_{1,2}-1=\sqrt{\frac{\sin ^{2} \delta_{1}}{Z_{1}} \frac{1}{Z_{2}}} \frac{\widetilde{U}_{1,2}}{\pi \Delta} .
\end{gathered}
$$

Among the three independent parameters of $R_{m, m^{\prime}}$ and $\widetilde{U}_{m, m^{\prime}}$, $R_{2,3}-1$ and $\widetilde{U}_{2,3}$ are for the doubly degenerate orbitals on the Fermi level. At zero field, $R_{2,3}$ and $\widetilde{U}_{2,3}$ take the SU(4) values $R_{2,3}-1=0.31$ and $\widetilde{U}_{2,3} /(\pi \Delta)=0.16$ for $U /(\pi \Delta)=$ 2.0 , and $R_{2,3}-1$ already approaches very closely to the value for the infinite Coulomb interaction: $R_{\mathrm{SU}(4)}^{\max }-1 \equiv 1 / 3$. These parameters continuously evolve from the SU(4) values to the SU(2) symmetric values: $R_{\mathrm{SU}(2)}-1=0.96$ and $\widetilde{U}_{2,3} /(\pi \Delta)=$ 0.23 .

We also discuss the field dependence of the other parameters, $R_{1,2}, R_{1,4}, \widetilde{U}_{1,2}$, and $\widetilde{U}_{1,4} . \widetilde{U}_{1,2}$ decreases from the SU(4) value with increasing magnetic field, and the corresponding Wilson ratio $R_{1,2}-1$ decreases to the noninteracting value 0 . In contrast to $\widetilde{U}_{1,2}, \widetilde{U}_{1,4}$ increases from the zero field value and becomes larger than $\widetilde{U}_{2,3}$ and $\widetilde{U}_{1,2}$ for $b>T_{K}^{\mathrm{SU}(4)}$. It further increases at the larger magnetic field regions $b \gg T_{K}^{\mathrm{SU}(4)}$ as shown in Fig. 4(f). This field dependence of $\widetilde{U}_{1,4}$ is similar to that of $\widetilde{U}$ for a single orbital Anderson model $[65,66]$, although $\widetilde{U}_{1,4}$ does not approach to the bare value $U$. We briefly discuss the field dependence of $\widetilde{U}$ and of the other Fermi liquid parameters for the single orbital Anderson model in Appendix D. This enhancement of $\widetilde{U}_{1,4}$ does not result in the enhancement of $R_{1,4}$. In fact, $R_{1,4}-1$ as well as $R_{1,2}-1$ decreases to 0 since the factor $\sin ^{2} \delta_{1}$ goes to 0 . We note that $R_{1,2}$ is slightly larger than $R_{1,4}$ at arbitrary $b$ in this case of $U /(\pi \Delta)=2.0$.

\section{B. Fermi-liquid parameters for larger $U$}

We next consider a strong coupling case, taking the Coulomb repulsion to be $U /(\pi \Delta)=4.0$, which is twice as large as the one studied in the above. For this case, effects of the interactions on the field-induced SU(4) to SU(2) Kondo crossover emerges in a pronounced way. Such a situation is also realistic because the experimental values of $U$ and $\Delta$ depend on individual quantum dots and on the valleys to be measured.

Figure 5(a) plots ground-state values of $\mathcal{T}_{m}(0)=\sin ^{2} \delta_{m}$ and of $\mathcal{M}_{14}$ as functions of magnetic fields $b / T_{K}^{\mathrm{SU}(4)}$ for both $U /(\pi \Delta)=4.0$ and $U /(\pi \Delta)=2.0$. The results for $U /(\pi \Delta)=4.0$ and $U /(\pi \Delta)=2.0$ are plotted with solid lines and dashed lines, respectively. The energy scale depends on the coupling constant as $T_{K}^{\mathrm{SU}(4)} / \Delta=0.2$ for $U /(\pi \Delta)=4.0$ and $T_{K}^{\mathrm{SU}(4)} / \Delta=0.41$ for $U /(\pi \Delta)=2.0$. We see that $\sin ^{2} \delta_{m}$ and $M_{d}$ of $U /(\pi \Delta)=4.0$ show almost same $b$ dependences as those of $U /(\pi \Delta)=2.0$, and thus they show the universality. The universal behavior is determined by the $b$ dependence of a single parameter $\delta_{1}\left(=\pi-\delta_{4}\right)$. Renormalized levels $\widetilde{\varepsilon}_{m}$ for $U /(\pi \Delta)=4.0$ plotted in Fig. 5(b) show the different $b$ dependence from those for $U /(\pi \Delta)=2.0$. Specifically, $\widetilde{\varepsilon}_{1}$ and $\widetilde{\varepsilon}_{4}$ stay closer to the Fermi level than those for $U /(\pi \Delta)=2.0$. However, this different $b$ dependence does not affect the universal behavior of $\delta_{1}$ because a ratio of $\widetilde{\varepsilon}_{m}$ to $\widetilde{\Delta}_{m}$ determines the phase shift $\delta_{m}$, i.e., $\delta_{m}=\cot ^{-1}\left(\widetilde{\varepsilon}_{m} / \widetilde{\Delta}_{m}\right)$.

Figures 5(c) and 5(d) show the renormalization factors $Z_{m}=\widetilde{\Delta}_{m} / \Delta$ and the Wilson ratios $R_{m, m^{\prime}}$, respectively. As in the $U /(\pi \Delta)=2.0$ case, the quasiparticle parameters $Z_{2}$ and $R_{23}$ for the doubly degenerate states at the Fermi level continuously evolve from the SU(4) value to the $\mathrm{SU}(2)$ value as $b$ varies from 0 to $\infty$. At zero field, these parameters take the SU(4) values: $Z_{\mathrm{SU}(4)}=0.25$ and $R_{\mathrm{SU}(4)}-1=0.33$ for $U /(\pi \Delta)=4.0$. Note that the Wilson ratio is almost saturated to the maximum possible value $R_{\mathrm{SU}(4)}^{\max }-1 \equiv 1 / 3$ at zero field. In the opposite limit $b \rightarrow \infty$, these parameters for the twofold degenerate states $(m=2,3)$ approach those for the symmetric $\mathrm{SU}(2)$ Anderson model: $Z_{\mathrm{SU}(2)} \rightarrow 0.026$ and $R_{23}^{\mathrm{SU}(2)}-1 \rightarrow 0.99$ for the same $U$. These results show that the strong Coulomb interaction significantly affects the renormalization factor $Z_{2}$ or $\widetilde{\Delta}_{2} . Z_{2}$ determines the energy scale for large field as $T_{K}^{\mathrm{SU}(2)}=0.02 \Delta$ with Eq. (45). The quasiparticle parameters $Z_{1}, R_{1,2}$ and $R_{1,4}$, for the states moving away from the Fermi level approach the noninteracting value in the limit of $b \rightarrow \infty$; i.e., $Z_{1} \rightarrow 1, R_{12} \rightarrow 1$, and $R_{14} \rightarrow 1$. Notably, $R_{1,4}$ becomes larger than $R_{1,2}$ for $U /(\pi \Delta)=4.0$ at finite $b$. This is quite different what we have found for the smaller interaction case $U /(\pi \Delta)=2.0$.

In order to clarify this difference, we plot the residual interactions $\widetilde{U}_{m, m^{\prime}}$ as functions of magnetic fields in Figs. 5(e) and 5(f). The magnetic fields of Figs. 5(e) and 5(f) are respectively scaled by $T_{K}^{\mathrm{SU}(4)}$ and $U$. In these figures, especially in Fig. 5(f), we can see that $\widetilde{U}_{1,4}$ becomes much larger than the other two residual interactions $\widetilde{U}_{1,2}$ and $\widetilde{U}_{2,3}$ as $b$ increases. This field dependence of $\widetilde{U}_{1,4}$ clearly explain why the corresponding Wilson ratio $R_{1,4}$ becomes larger than $R_{1,2}$. We also note that $\widetilde{U}_{2,3}$ for the doubly degenerate levels approach the $\mathrm{SU}(2)$ symmetric value $\widetilde{U}_{2,3} /(\pi \Delta) \rightarrow 0.026$.

All these results discussed in this section indicate that the quantum fluctuations and many-body effects are enhanced for large magnetic fields as the number of active channel decreases from 4 to 2 [36]. We have also shown the enhancement of the fluctuations are more clearly seen for strong interactions by comparing the results for $U /(\pi \Delta)=4.0$ to those for $U /(\pi \Delta)=2.0$.

\section{TEMPERATURE DEPENDENCE OF MAGNETOCONDUCTANCE}

The above discussions about the Fermi-liquid parameters have mainly focused on the zero temperature properties of the 

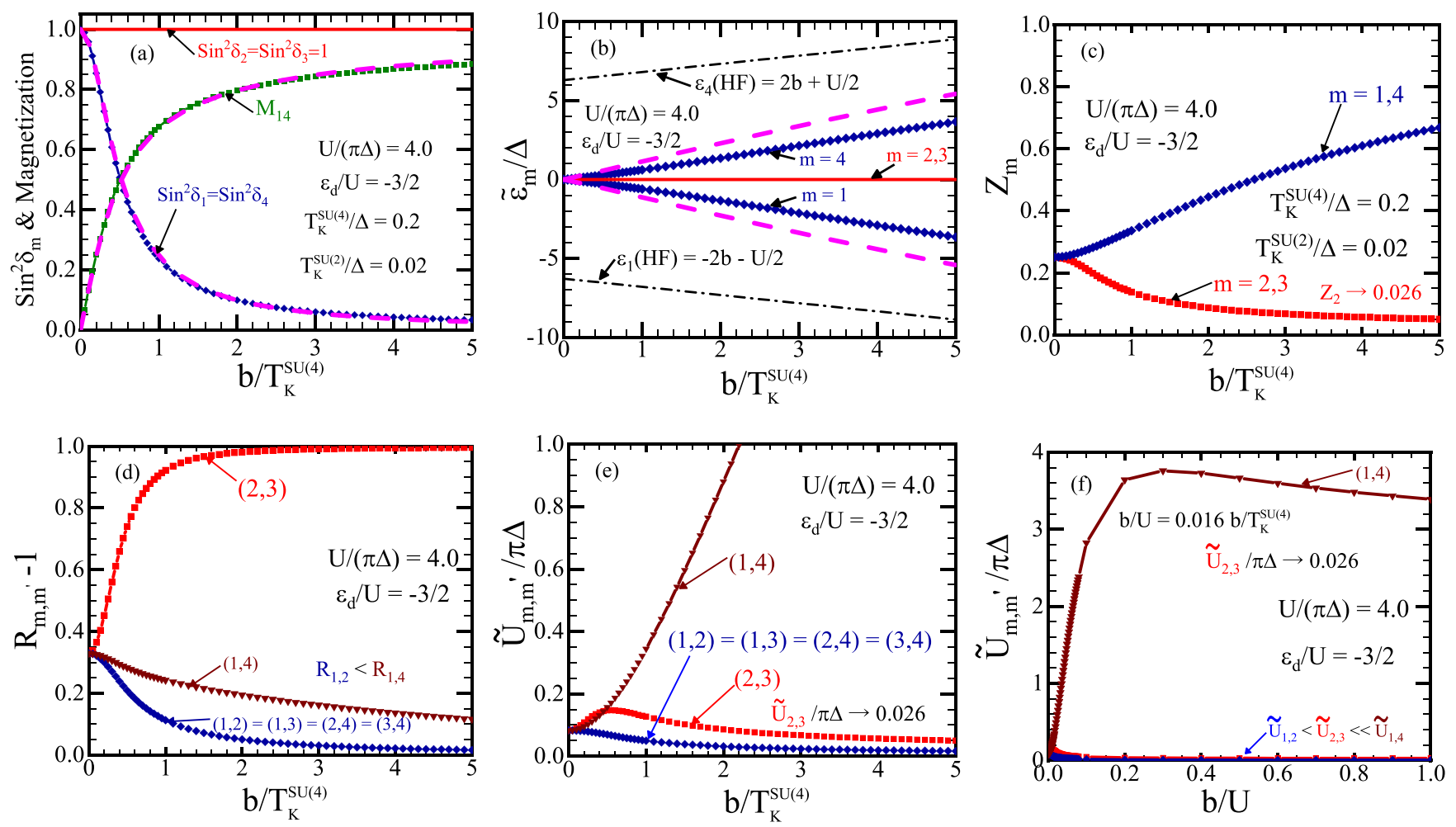

FIG. 5. (a) $\sin ^{2} \delta_{m}$ and magnetization $\mathcal{M}_{14}=\left\langle n_{d 1}\right\rangle-\left\langle n_{d 4}\right\rangle$, (b) renormalized level position $\widetilde{\varepsilon}_{m}$, (c) renormalization factor $Z_{m}$, (d) Wilson ratio $R_{m, m^{\prime}}-1$, and (e) residual interaction $\widetilde{U}_{m, m^{\prime}}$ are plotted as functions of magnetic field $b$ at half-filling $\varepsilon_{d} / U=-3 / 2$ for $U /(\pi \Delta)=4.0$. The $\mathrm{x}$ axis in (a)-(e) is scaled by the SU(4) Kondo temperature $T_{K}^{\mathrm{SU}(4)}=0.2 \Delta=(0.016 U)$ determined at $b=0$. The axis in (f) is scaled by $U$ for examining the behavior of $\widetilde{U}_{m, m^{\prime}}$ at larger magnetic fields. The dot levels $\epsilon_{m}$ are chosen in a such way that is described in Eq. (41). In (a), $\sin ^{2} \delta_{m}$ and $M_{d}$ for $U /(\pi \Delta)=2.0$ are also plotted by dashed lines to compare them with those for the present case $U /(\pi \Delta)=4.0$. Similarly, $\widetilde{\varepsilon}_{m}$ for $U /(\pi \Delta)=2.0$ are plotted in (b). The dash-dotted lines indicate the mean-field splitting $\varepsilon_{1}^{\mathrm{HF}}=-(2 b+U / 2)$ and $\varepsilon_{4}^{\mathrm{HF}}=(2 b+U / 2)$. In the limit of $b \rightarrow \infty, Z_{2}, R_{2,3}-1$, and $\tilde{U}_{2,3}$ approach the $\mathrm{SU}(2)$ values for $U /(\pi \Delta)=4.0: Z_{2} \rightarrow 0.026, R_{2,3} \rightarrow 1.99$, and $\widetilde{U}_{2,3} / \pi \Delta \rightarrow 0.026$.

crossover. The results show that the quasiparticles are strongly renormalized as the ground state undergoes the crossover from the SU(4) Kondo state to the SU(2) Kondo state.

We also study finite temperature properties of the crossover in this section by calculating each component of the magnetoconductance $g_{m}$ for $m=1,2,3,4$ and the total conductance $g_{\text {tot }}$. At half-filling, $\varepsilon_{d}=-\frac{3}{2} U$, only two components are independent: $g_{2}=g_{3}$ and $g_{1}=g_{4}$ due to the level structure described in Eq. (41). The finite-temperature conductance, defined in Eq. (23), depends on the excited states whose contributions enter through the spectral function $A_{m}(\omega, T)$. We calculate the $T$-dependent $A_{m}(\omega, T)$, using the NRG with some extended methods for dynamic correlation functions described in Sec. II D and Appendix E, to obtain $g_{m}$. We examine two different interactions, $U /(\pi \Delta)=2.0$ and 4.0, also for these components of the conductance assuming symmetric couplings $\Delta_{L}=\Delta_{R}=\Delta / 2$.

\section{A. Conductance for $U /(\pi \Delta)=2.0$ at half-filling}

Figures 6(a)-(d) plot the total conductance $g_{\text {tot }}$ and the components $g_{2}=g_{3}$ and $g_{1}=g_{4}$ as functions of the temperature for six values of magnetic fields, $b / T_{K}^{\mathrm{SU}(4)}=$ $0.0,0.25,0.5,1.0,2.0$, and 4.0. The SU(4) Kondo energy scale, determined at $b=0$ for $U /(\pi \Delta)=2.0$, is estimated to be $T_{K}^{\mathrm{SU}(4)}=0.41 \Delta$ as mentioned in Sec. IV A.
Figure 6(a) shows that the total counductance at $b=0$ logarithmically increases around $T \sim T_{K}^{\mathrm{SU}(4)}$. This logarithmic temperature dependence is a hallmark of the SU(4) Kondo effect. $g_{\text {tot }}$ increases to the unitary-limit value $4 e^{2} / h$ as temperature goes down to $T \rightarrow 0$. As the magnetic fields increase, the conductance at low-temperatures $T \ll T_{K}^{\mathrm{SU}(2)}$ decreases from the SU(4) unitary limit value $4 e^{2} / h$ to the $\mathrm{SU}(2)$ one $2 e^{2} / h$. We can also see that in a temperature range of $0.1 T_{K}^{\mathrm{SU}(2)} \lesssim T \lesssim T_{K}^{\mathrm{SU}(4)}$ the conductance curve deforms continuously into the curve for the SU(2) symmetric case. The $\mathrm{SU}(2)$ Kondo state emerges for $b \rightarrow \infty$ where the characteristic energy scale becomes $T_{K}^{\mathrm{SU}(2)}=0.19 \Delta$. Therefore the finite temperature conductance also shows the crossover behavior. To examine how the magnetoconductance $g_{\text {tot }}$ evolves with increasing $b$ in more detail, we discuss the two components, $g_{2}$ and $g_{1}$.

Figure 6(b) plots the component $g_{2}$ which represents contributions of the state $m=2$ and $m=3$ remaining at the Fermi level. This component $g_{2}$ decreases as $b$ increases in the temperature range of $0.1 T_{K}^{\mathrm{SU}(2)} \lesssim T \lesssim T_{K}^{\mathrm{SU}(4)}$. In order to investigate the behavior of $g_{2}$ in this range in more detail, we introduce an energy scale $T_{2}^{*}$ :

$$
T_{2}^{*} \equiv \frac{\pi}{4} \widetilde{\Delta}_{2}, \quad \widetilde{\Delta}_{2}=Z_{2} \Delta
$$



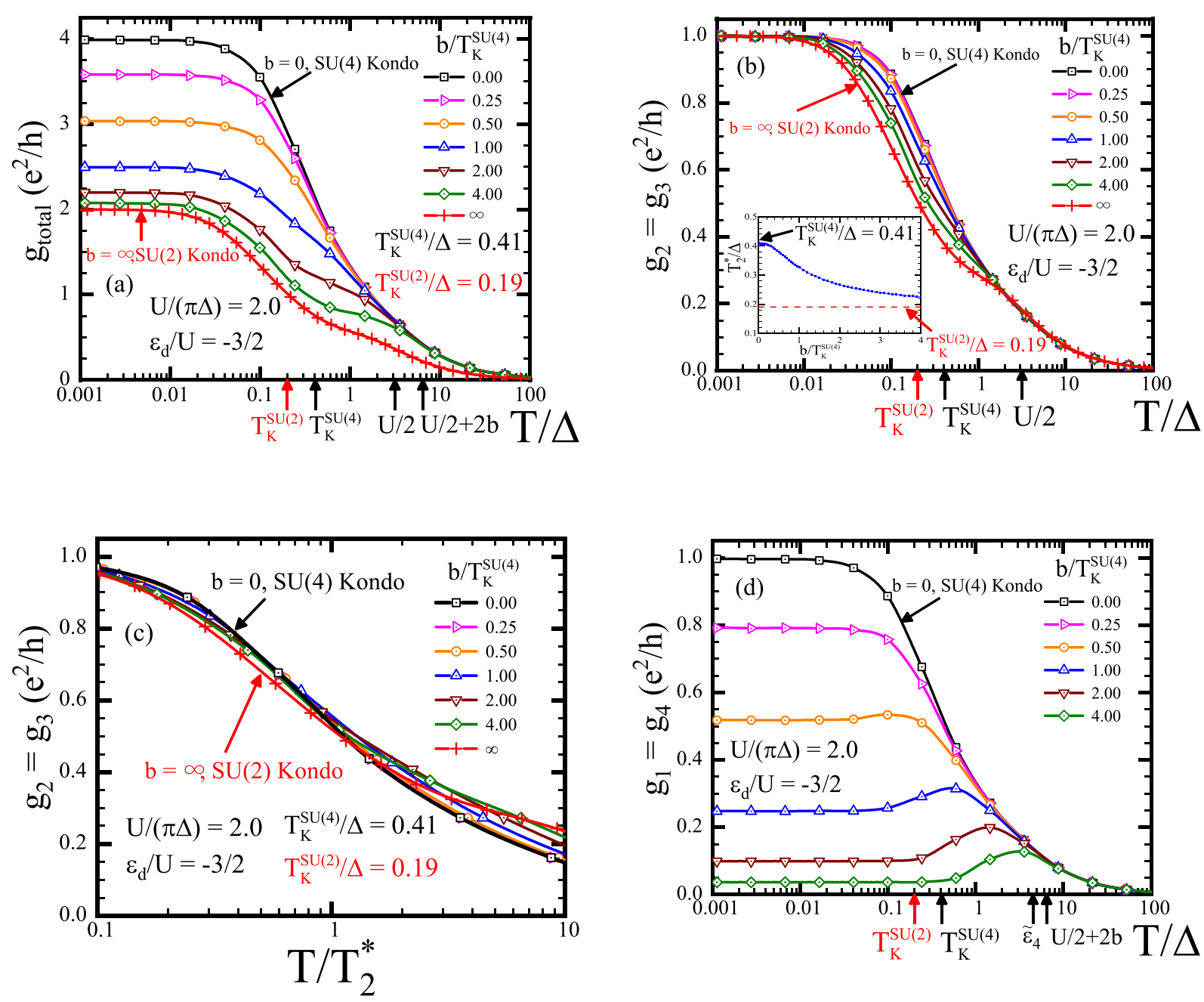

FIG. 6. Temperature dependence of the linear conductance for $U /(\pi \Delta)=2.0$ are plotted for six values of magnetic fields $b / T_{K}^{\mathrm{SU}(4)}=$ $0.0,0.25,0.5,1.0,2.0$, and 4.0 at half-filling $\varepsilon_{d}=-3 U / 2$. (a) shows the total conductance $g_{\text {tot }}=\sum_{m=1}^{4} g_{m}$. The conductance consists of two components, i.e., $g_{2}=g_{3}$ and $g_{1}=g_{4}$. (b) and (c) show the first one $g_{2}$, and (d) shows the second one $g_{1}$. (a)-(c) also show the results for the SU(2) symmetric case by the symbols (+). In (a), (b), and (d), the $x$ axis is normalized the bare resonance width $\Delta$, and the axis in (c) is normalized by a field dependent energy scale $T_{2}^{*}=(\pi / 4) Z_{2} \Delta$. The inset of (b) shows $T_{2}^{*}$ as functions of $b / T_{K}^{\mathrm{SU}(4)}$. At $b=0, T_{2}^{*}$ takes the SU(4) symmetric value, $T_{K}^{\mathrm{SU}(4)} / \Delta=0.41$. In the opposite limit $b=\infty$, it takes $\mathrm{SU}(2)$ value, $T_{K}^{\mathrm{SU}(2)} / \Delta=0.19$ which is indicated by the dashed line. The vertical arrows at the bottom of the panels indicate $T_{K}^{\mathrm{SU}(2)}, T_{K}^{\mathrm{SU}(4)}, U / 2, \widetilde{\varepsilon}_{4}$ and $U / 2+2 b$; specifically the last two, $\varepsilon_{4}$ and $U / 2+2 b$, are defined with respect to $b / T_{K}^{\mathrm{SU}(4)}=4.0$.

Aside from a numerical factor $\pi / 4$, this energy $T_{2}^{*}$ corresponds to the width $\widetilde{\Delta}_{2}\left(=\widetilde{\Delta}_{3}\right)$ of the Kondo resonance for $m=2$ and 3, locked at the Fermi level even at finite magnetic fields. We use this energy scale to examine the scaling behavior. $T_{2}^{*}$ coincides with $T_{K}^{\mathrm{SU}(4)}=0.41 \Delta$ at $b=0$, and with $T_{K}^{\mathrm{SU}(2)}=0.19 \Delta$ in the opposite limit $b \rightarrow \infty$. The inset of Fig. 6(b) shows the energy scale $T^{*}$ as functions of $b$. We can see that $T_{2}^{*}$ decreases from $T_{K}^{\mathrm{SU}(4)}$ to $T_{K}^{\mathrm{SU}(2)}$ with increasing $b$. Correspondingly, a region where $g_{2}$ shows the $\log T$ dependence moves towards a low temperature side as $b$ increases. Although $g_{2}$ decreases with increasing $b$ at the finite temperatures, it approaches the unitary limit $e^{2} / h$ for $T \rightarrow 0$ for arbitrary magnetic fields. This is because the matching of the spin and orbital Zeeman splitting locks the phase shifts at $\delta_{2}=\delta_{3}=\pi / 2$ even for magnetic fields.
Another important aspect of the crossover is the scaling behavior of the conductance. In Ref. [28], Mantelli and his coworkers examine effects of the spin-orbit interaction on the scaling behavior at quarter filling, $\varepsilon_{d}=-\frac{1}{2} U$. We examine how the magnetic field affects the scaling at half-filling, $\varepsilon_{d}=-\frac{3}{2} U$. To explore the scaling behavior, we rescale temperatures by the field dependent energy scale $T_{2}^{*}$ defined in Eq. (57) and replot $g_{2}$ for the six values of $b$ as functions of the rescaled temperatures $T / T_{2}^{*}$ in Fig. 6(c). The six curves are almost overlapped each other in this figure. At low fields $b \lesssim T_{K}^{\mathrm{SU}(4)}$, the curves remain the same curve as the $\mathrm{SU}(4)$ universal one over a wide temperature range. At high fields $b \gg T_{K}^{\mathrm{SU}(4)}$, the curves collapse into the $\mathrm{SU}(2)$ universal curve. At half-filling points $\epsilon_{d}=-\frac{N-1}{2} U$, the Wilson ratio determines the $T^{2}$ coefficient $C_{T}$ for the $\mathrm{SU}(N)$ 
conductance: $C_{T}=\left(\pi^{2} / 48\right)\left[1+2(N-1)(R-1)^{2}\right]$. Substituting the Wilson ratio for the $\mathrm{SU}(4)$ case $R^{\mathrm{SU}(4)}-1=0.32$ and for the $\mathrm{SU}(2)$ case $R^{\mathrm{SU}(2)}-1=0.96$ into the formula of $C_{T}$ yields the $T^{2}$ coefficients $C_{T}$ of each case for $U /(\pi \Delta)=$ 2.0: $C_{T}^{\mathrm{SU}(4)} \simeq 0.33$ and $C_{T}^{\mathrm{SU}(2)} \simeq 0.59$. Since $C_{T}^{\mathrm{SU}(4)}<C_{T}^{\mathrm{SU}(2)}$, the conductance for $N=4$ is larger than that for $N=2$ at the low-temperature regions $T / T_{2}^{*} \lesssim 0.1$. Figure 6(c) shows this magnitude relation of the conductance, and thus demonstrates that the scaling behavior depends on the number of orbitals $N$ and the Wilson ratio $R$.

Figure 6(d) shows the other component $g_{1}\left(=g_{4}\right)$ which correspond to the contributions of the other two state moving away from the Fermi level. At low temperatures $T \lesssim T_{K}^{\mathrm{SU}(4)}$, these components decrease as $b$ increases and eventually vanish at the high magnetic fields $b \gg T_{K}^{\mathrm{SU}(4)}$. We can also see that $g_{1}$ has a peak at large mange fields $b \lesssim 0.5 T_{K}^{\mathrm{SU}(4)}$. The emergent peak is caused by thermal excitations from (to) the renormalized level $\widetilde{\varepsilon}_{1}\left(\widetilde{\varepsilon}_{4}\right)$ which situates deep inside (far above) the Fermi level for large fields as shown in Fig. 5(b). Furthermore, tor the large fields, the level structure of the CNT dot approaches the mean-field levels described in Eq. (53), and the atomic-limit peak also emerges at $U / 2+2 b$ (see also Appendix F).

These results obtained for $U /(\pi \Delta)=2.0$ show a rather moderate evolution of the crossover and the Kondo energy scale $T_{2}^{*}$ as $T_{K}^{\mathrm{SU}(2)}$ is only half of $T_{K}^{\mathrm{SU}(4)}$.

\section{B. Conductance for $U /(\pi \Delta)=4.0$ at half-filling}

We next examine the conductance for a case of strong interaction $U /(\pi \Delta)=4.0$ to see more clearly the field-induced crossover at finite temperatures. In this case, the characteristic energy scale for the $\mathrm{SU}(2)$ case is significantly suppressed $T_{K}^{\mathrm{SU}(2)}=0.02 \Delta$, which becomes much smaller than the $\mathrm{SU}(4)$ energy scale $T_{K}^{\mathrm{SU}(4)}=0.2 \Delta$, i.e., the difference is about one order of magnitude.

Figure 7(a) shows that the total conductance $g_{\text {tot }}$ for a temperature region $0.1 T_{K}^{\mathrm{SU}(2)} \lesssim T \lesssim T_{K}^{\mathrm{SU}(4)}$ decreases as magnetic field increases. Since the characteristic energy scale $T_{2}^{*}$ defined in Eq. (57) in this case becomes much smaller than that for $U /(\pi \Delta)=2.0$, the region where the crossover occurs moves towards a low-temperature region, $T \lesssim T_{K}^{\mathrm{SU}(4)}=$ $0.2 \Delta$. Furthermore, the shoulder structures emerging in the high temperature region are more pronounced.

Figure 7(b) clearly shows that the curves of $g_{2}$ evolve from the SU(4) curve to the $\mathrm{SU}(2)$ curve during the crossover. Specifically, the energy scale $T_{2}^{*}$ around which $g_{2}$ shows $\log T$ dependence decreases with increasing magnetic field. The inset of Fig. 6(b) shows the suppression of $T_{2}^{*}$ : it decreases from $T_{K}^{\mathrm{SU}(4)}=0.2 \Delta$ to $T_{K}^{\mathrm{SU}(2)}=0.02 \Delta$.

The scaling behavior of $g_{2}$ in Fig. 6(c) also becomes clear because of this suppression. In a wide range of temperatures, we can see that the scaled results collapse into two different universal curves, i.e., the SU(4) curve for small fields $b / T_{K}^{\mathrm{SU}(4)} \lesssim 0.25$, and $\mathrm{SU}(2)$ curve for large fields $b / T_{K}^{\mathrm{SU}(4)} \gtrsim$ 1. Since the Wilson ratios for $N=4$ and 2 are respectively saturated to the maximum possible values $R_{\mathrm{SU}(4)}^{\max }-1=1 / 3$ and $R_{\mathrm{SU}(2)}^{\max }-1=1$, the $T^{2}$ coefficients $C_{T}$ for each $N$ are also saturated: $C_{T}^{\mathrm{SU}(4)} \simeq 0.34$ and $C_{T}^{\mathrm{SU}(2)} \simeq 0.62$. Figure 6(c) clearly shows that $g_{2}$ for $b=0$ is larger than that for $b \rightarrow \infty$ at $T<T_{2}^{*}$ because of $C_{T}^{\mathrm{SU}(4)}<C_{T}^{\mathrm{SU}(2)}$.

Furthermore, we can recognize that a broad peak emerges for $b \gg T_{K}^{\mathrm{SU}(4)}$ at $T \approx U / 2$. It corresponds to an thermal energy needed to add an electron or a hole to the degenerate states. Thus this atomic limit peak and the quasiparticle excitation peak in Fig. 7(d) at $T \approx 2 b+U / 2$ yield the shoulder structure of $g_{\text {tot }}$ at the high temperatures, as shown in Fig. 7(a).

\section{SPECTRAL PROPERTIES ALONG THE FIELD-INDUCED CROSSOVER}

Spectral functions at finite magnetic fields also reflect the crossover from the SU(4) to SU(2) Kondo states. In addition to the Kondo resonance near the Fermi level, the Zeemann splitting causes a shift of the atomic-limit peak to $\pm(2 b+U / 2)$. The spectral functions for the doubly degenerate states remain the same $A_{2}(\omega, T)=A_{3}(\omega, T)$ for finite magnetic fields owing to the dot level structure given in Eq. (41). Following relations additionally hold in the particle-hole symmetric case $\varepsilon_{d}=-(3 / 2) U$,

$$
A_{2}(\omega, T)=A_{2}(-\omega, T), \quad A_{1}(\omega, T)=A_{4}(-\omega, T) .
$$

The second relation shows that $A_{4}$ is a mirror image of $A_{1}$, and we discuss $A_{1}$ and $A_{2}$ in the following. We examine the spectral functions at $T=0$, and hence we drop the second argument of the functions, namely, $A_{m}(\omega) \equiv A_{m}(\omega, T=$ $0),(m=1,2,3,4)$. As in the previous sections, we consider the two cases for the interaction: (i) $U /(\pi \Delta)=2.0$ and (ii) $U /(\pi \Delta)=4.0$. The spectral function obtained by the NRG procedure is a set of discrete $\delta$ functions. To obtain a continuous spectrum, the logarithmic Gaussian function is used (see Appendix E 3).

\section{A. Spectral function for $U /(\pi \Delta)=\mathbf{2 . 0}$}

Figure 8(a) shows the total spectral function $A_{\text {tot }}(\omega)=$ $\sum_{m=1}^{4} A_{m}(\omega)$ for five different values of magnetic fields and $U /(\pi \Delta)=2.0$. At zero magnetic field, we can see that a single SU(4) Kondo resonance peak emerges on the Fermi level $\omega=0$. As the magnetic field increases in the range of $0<b<\infty$, the height of the Kondo peak decreases from 4 to 2 in units of $\pi \Delta$ since the resonance peak positions for $m=1$ and 4 move away from the Fermi level, leaving the other positions for $m=2$ and 3 just on the Fermi level, as shown in Fig. 4(a). This field dependence of the peak positions results in deforming the peak shape of the SU(4) Kondo resonance into that of the SU(2) Kondo resonance on the Fermi level. Furthermore, two sub peaks emerge at higher energies, i.e., $\pm(2 b+U / 2)$.

Figure 8(b) plots $A_{2}\left(=A_{3}\right)$ for four values of $b . A_{2}\left(=A_{3}\right)$ shows such deformation of the peak shape. The deformation of $A_{2}$ is not so clear in the case of $U /(\pi \Delta)=2.0$ since $T_{K}^{\mathrm{SU}(2)}$ is only half as large as $T_{K}^{\mathrm{SU}(4)}$. Nevertheless, the inset of Fig. 8(b) which is an enlarged view around the Fermi level shows that the resonance width on the Fermi level becomes sharper. The sharpening of the width leads to the decrease of the spectral weight. As the magnetic field increases, $A_{2}$ also develops two sub peaks at $\omega= \pm U / 2$ to compensate the decrease of the weight. The two peak positions correspond to 

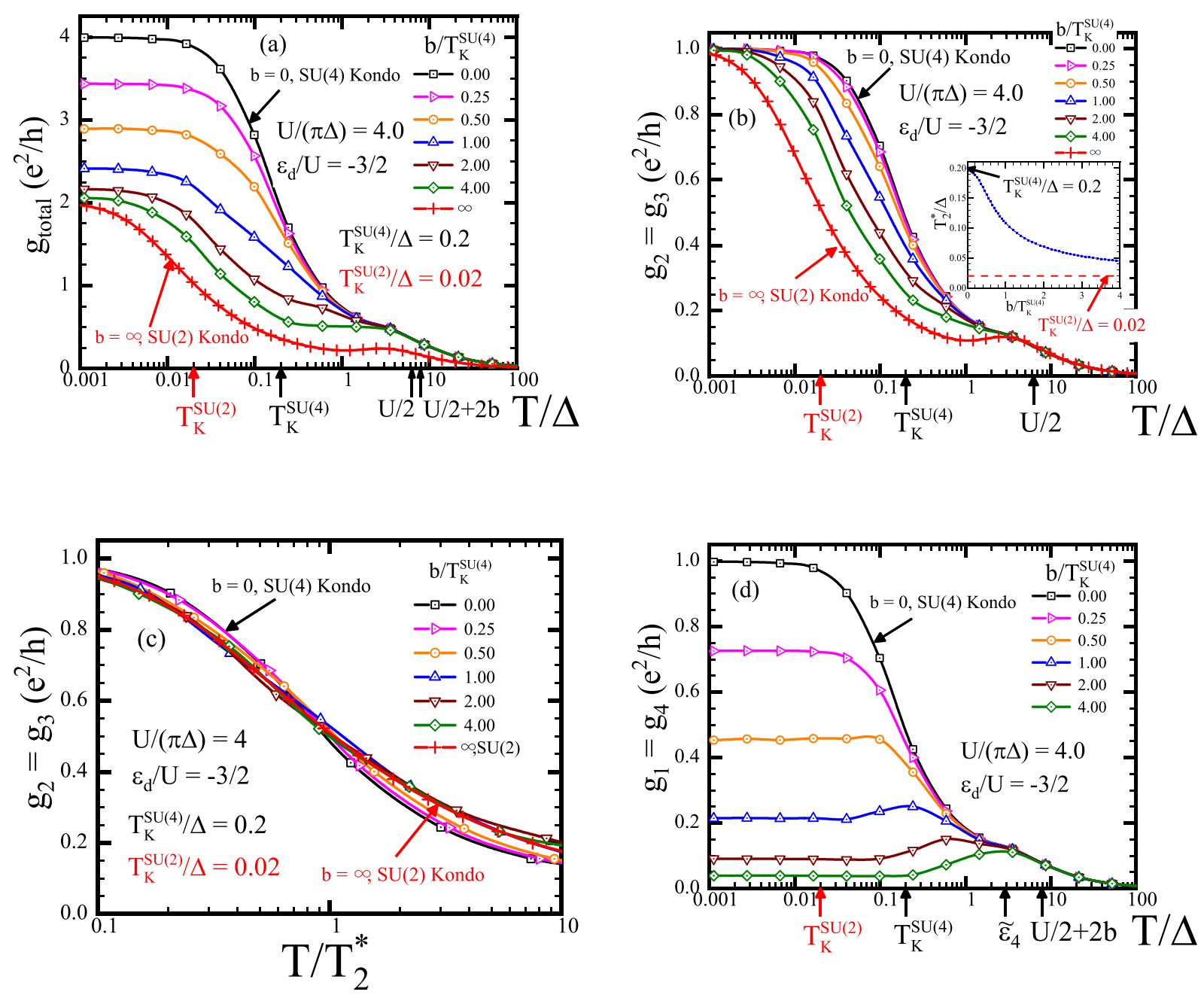

FIG. 7. Temperature dependence of the linear conductance for $U /(\pi \Delta)=4.0$ are plotted for six values of magnetic fields $b / T_{K}^{\mathrm{SU}(4)}=$ $0.0,0.25,0.5,1.0,2.0,4.0$ at half-filling $\varepsilon_{d}=-3 U / 2$. (a) shows the total conductance $g_{\text {tot }}=\sum_{m=1}^{4} g_{m}$. The conductance consists of two components, i.e., $g_{2}=g_{3}$ and $g_{1}=g_{4}$. (b) and (c) show the first one $g_{2}$, and (d) shows the second one $g_{1}$. (a)-(c) also show the results for the SU(2) symmetric case by the symbols (+). In (a), (b), and (d), the $\mathrm{x}$ axis is normalized the bare resonance width $\Delta$, and the axis in (c) is normalized by characteristic energy scales $T_{2}^{*}$. The inset of (b) shows $T_{2}^{*}$ as functions of $b / T_{K}^{\mathrm{SU}(4)}$. At $b=0, T_{2}^{*}$ takes the SU(4) symmetric value, $T_{K}^{\mathrm{SU}(4)} / \Delta=0.20$. In the opposite limit $b=\infty$, it takes $\mathrm{SU}(2)$ value, $T_{K}^{\mathrm{SU}(2)} / \Delta=0.02$ which is indicated by the dashed line. The vertical arrows at the bottom of the panels indicate $T_{K}^{\mathrm{SU}(2)}, T_{K}^{\mathrm{SU}(4)}, U / 2, \widetilde{\varepsilon}_{4}$, and $U / 2+2 b$; specifically the last two, $\varepsilon_{4}$ and $U / 2+2 b$, are defined with respect to $b / T_{K}^{\mathrm{SU}(4)}=4.0$.

the excitation energies on adding an electron or hole to the dot. In Appendix F, we provide analytic expressions of the spectral functions in the atomic limit $v_{v} \rightarrow 0$, where the CNT dot is disconnected from the metallic leads. The remaining degenerate states turn into the SU(2) Kondo state in the limit of $b \rightarrow \infty$. indicating that the states undergo the crossover from the SU(4) to SU(2) Kondo state.

Figure 8(c) shows the other component $A_{1}(\omega)$, which corresponds to the component of the level going down from the Fermi level. Note that $A_{4}(\omega)=A_{1}(-\omega)$, as mentioned. We can see that the spectral weight transfers to the negative frequency region $\omega<0$, and an evolution of its resonance peak position shows good agreement with the field dependence of $\widetilde{\varepsilon}_{1}$ presented in Fig. 4(a). This transfer leads to the development of the sub peaks and decrease of the Kondo peak of $A_{\text {tot }}$. With increasing magnetic fields, the resonance peak at $\omega=\widetilde{\varepsilon}_{1}$ merges with the atomic-limit peak at $\omega=-U / 2-2 b$, which shifts from the zero field position $-U / 2$ in the presence of $b$. This shift of the atomic-limit peak, which we discuss in the Appendix F, results from the descent of the energy level $\varepsilon_{1}$ described in Eq. (41). For much larger fields $b \gg T_{K}^{\mathrm{SU}(4)}$, the curve of $A_{1}$ approaches the Lorentzian form. Therefore the quasiparticle state of $m=1$ is unrenormalized from the correlated Kondo state to the bare state.

\section{B. Spectral function for $U /(\pi \Delta)=\mathbf{4 . 0}$}

In order to investigate the effects of the strong interaction on the spectral functions, we next discuss the spectral functions for $U /(\pi \Delta)=4$.0. Figures 9(a)-9(c) respectively show results of $A_{\text {tot }}, A_{2}$, and $A_{1}$. We compare these results with the corresponding results for the weak interaction case in Fig. 8, $A_{\text {tot }}$ for the strong interaction in Fig. 9(a) shows a similar trend as that for $U /(\pi \Delta)=2.0$ in Fig. 8(a). However, 

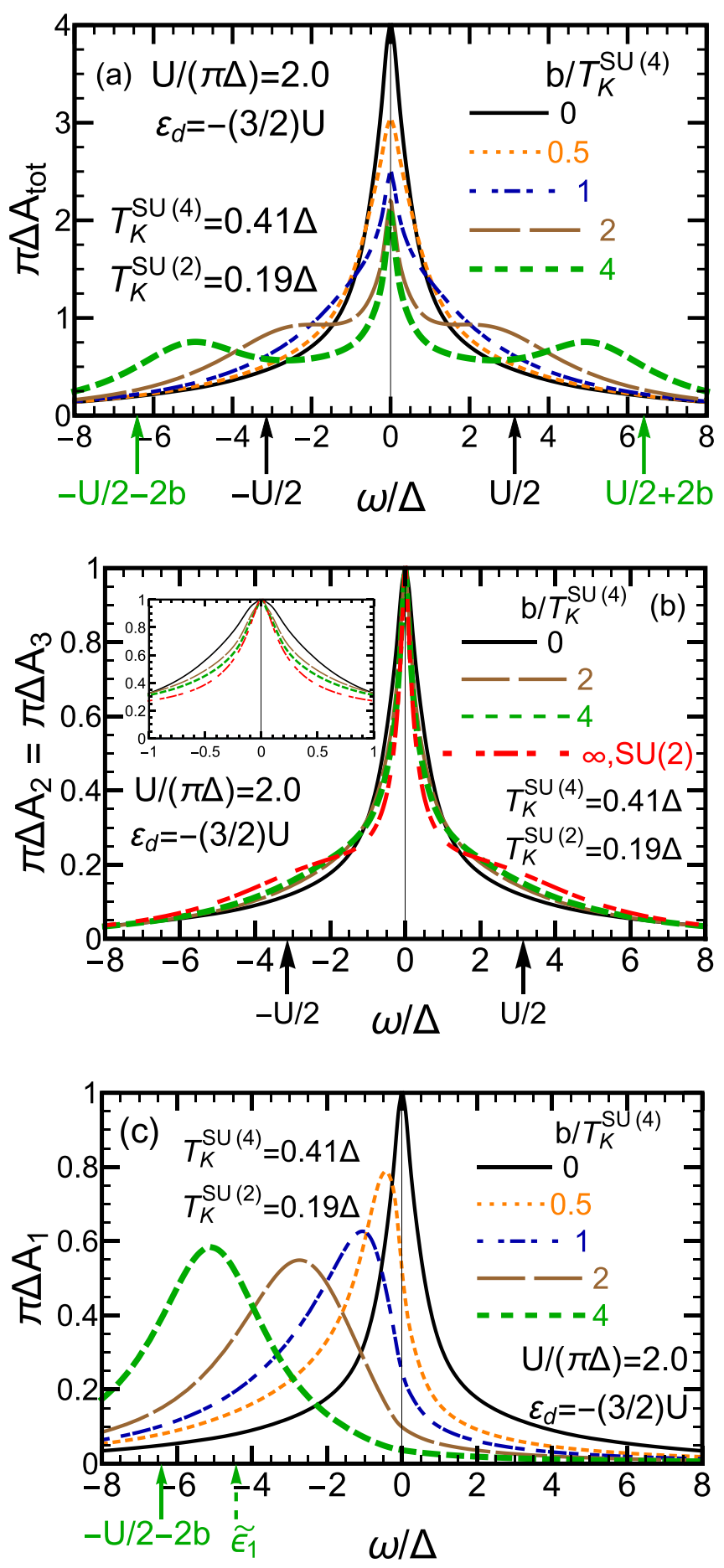

FIG. 8. Zero temperature spectral functions for $U /(\pi \Delta)=$ 2.0 are plotted for five values of magnetic fields, $b / T_{K}^{\mathrm{SU}(4)}=$ $0.0,0.5,1.0,2.0,4.0$ at half-filling $\varepsilon_{d}=-3 U / 2$ : (a) $A_{\text {tot }}(\omega)=$ $\sum_{m=1}^{4} A_{m}(\omega)$, (b) $A_{2}(\omega)$, and (c) $A_{1}(\omega)$. Vertical arrows at the bottom of the panels indicate the points $\omega= \pm U / 2$ and $\pm(2 b+U / 2)$ where peaks emerge in the atomic limit. The peaks of $\omega= \pm(2 b+U / 2)$ are for the largest value of $b$ among the five, $b / T_{K}^{\mathrm{SU}(4)}=4.0$. The position of the renormalized resonance level $\widetilde{\varepsilon}_{1}$ for the same value of $b$ are also shown in the bottom.

the width of resonance for $U /(\pi \Delta)=4.0$ is smaller than that for $U /(\pi \Delta)=2.0$ in arbitrary magnetic fields, because $U$ is larger.
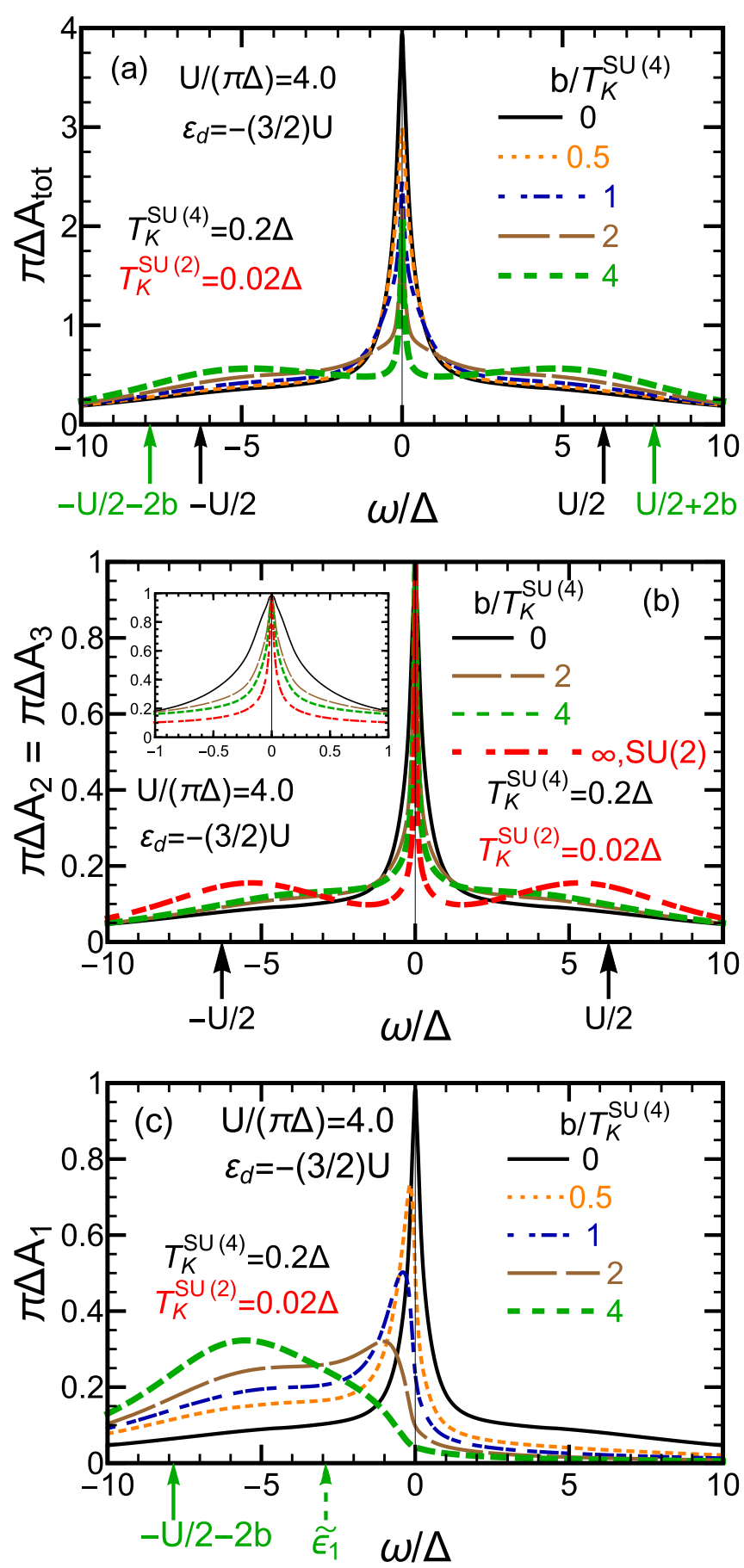

FIG. 9. Spectral functions for $U /(\pi \Delta)=4.0$ are plotted for five values of magnetic fields, $b / T_{K}^{\mathrm{SU}(4)}=0.0,0.5,1.0,2.0,4.0$ at half-filling $\varepsilon_{d}=-3 U / 2$ : (a) $A_{\text {tot }}(\omega)=\sum_{m=1}^{4} A_{m}(\omega)$, (b) $A_{2}(\omega)$, and (c) $A_{1}(\omega)$. Vertical arrows at the bottom of the panels indicate the points $\omega= \pm U / 2$ and $-(2 b+U / 2)$ where peaks emerge in the atomic limit. The peaks of $\omega= \pm(2 b+U / 2)$ are for the largest value of $b$ among the five, $b / T_{K}^{\mathrm{SU}(4)}=4.0$. The position of the renormalized resonance level $\widetilde{\varepsilon}_{1}$ for the same value of $b$ are also shown in the bottom.

The component $A_{2}$ in Fig. 9(b) more clearly shows the narrowing of the resonance width than that for $U /(\pi \Delta)=2.0$, because $T_{K}^{\mathrm{SU}(2)}$ is smaller than $T_{K}^{\mathrm{SU}(4)}$ by one order of magnitude in this case i.e., $T_{K}^{\mathrm{SU}(2)}=0.02 \Delta$ and $T_{K}^{\mathrm{SU}(4)}=0.2 \Delta$. 
The narrowing of the width leads to a loss of the spectral weight around the Fermi level, which is compensated by an enhancement of the atomic-limit peak at $\pm U / 2$.

The atomic limit peak around $-U / 2-2 b$ of $A_{1}$ is broader in the strong interaction case shown in Fig. 9(c) than in the weak interaction case, because the quasiparticle resonance position $\widetilde{\varepsilon}_{1}$ presented in Fig. 5(b) still remains around the Fermi level at the higher fields, $b \gg T_{K}^{\mathrm{SU}(4)}$. Owing to this remaining, the quasiparticle state is still renormalized even at the higher fields, and thus the shape of $A_{1}$ differs from the Lorentzian form.

\section{SUMMARY}

We have studied the Kondo effect in a carbon nanotube quantum dot in a wide range of temperature and magnetic field using the numerical renormalization group.

In the first half of the present paper, we have studied finite temperature properties of the SU(4) Kondo state by calculating the finite temperature conductance in a wide range of electron filling $N_{d}$. The NRG results nicely agree with the experimental results in the wide range, supporting an emergence of the SU(4) Kondo resonance at low-temperatures, $T \lesssim T_{K}^{\mathrm{SU}(4)}$. Furthermore, we have precisely examined the temperature dependence of conductance especially at two fixed values of $N_{d}$ : quarter-filling $N_{d}=1$ and half-filling $N_{d}=$ 2. The obtained results show that the scaled conductance of $N_{d}=1$ is larger than that of $N_{d}=2$ at the low temperatures. A microscopic Fermi-liquid theory, which is extended to arbitrary $N_{d}$, successfully explains such different behavior of the conductance depending on $N_{d}$. The theory shows that a $T^{2}$ coefficient $C_{T}$ for the conductance vanishes at $N_{d}=1$. In contrast to the quarter-filling case, $C_{T}$ does not become zero at half-filling, but saturates to a strong coupling limit value. Thus the universality depends on the electron filling. We expect that this filling dependence of the universality can be observed.

In the second half of the present paper, we have investigated how magnetic fields affect the ground state and also excited states in the course of the SU(4) to SU(2) Kondo crossover. Our previous papers show that quasiparticle states remaining the Fermi level are renormalized as the number of active levels decreases from four to two. The other two states become unrenormalized in the course of the crossover. The present paper has shown that the renormalization of the quasiparticle states more clearly appear in a strong interaction case because a characteristic energy scale $T_{2}^{*}$ clearly decreases from the SU(4) Kondo energy scale $T_{K}^{\mathrm{SU}(4)}$ to the $\mathrm{SU}(2)$ Kondo energy scale $T_{K}^{\mathrm{SU}(2)}$.

The finite temperature conductance in the magnetic fields also shows such decrease of the energy scale. In addition, the scaling behavior at half-filling shows that the excited states undergo the crossover. Specifically, as soon as the magnetic fields $b$ become comparable to $T_{K}^{\mathrm{SU}(4)}$, the SU(4) universality is lost, and for the much larger fields, $b \gg T_{K}^{\mathrm{SU}(4)}$, the $\mathrm{SU}(2)$ universality emerges. Furthermore, the NRG results for both $\mathrm{SU}(2)$ and SU(4) symmetric cases indicate that the Wilson ratio and the Kondo energy scale determine the low-temperature behavior of half-filled quantum dots.
We have also calculated total spectral function and their components in magnetic fields. The obtained spectral function shows that the resonance states remaining on the Fermi level become sharper as the magnetic field increases, showing a good agreement with the field dependence of the corresponding renormalized resonance width. Furthermore, a spectral weight of the other two states transfers towards the higher frequency region, because the Zeeman splitting shifts the two peak positions upward and downward from the Fermi level. Such transfer results in the emergence of two sub-peaks whose positions approach atomic-limit peak positions.

An interesting future work is to explore how the thermoelectric transport of multilevel quantum dots depends on the electron filling $[42,67,68]$. We have already had the lowtemperature expansions for the thermal conductance [34], and the progress along this line will be discussed elsewhere.

\section{ACKNOWLEDGMENTS}

This work was supported by Grant-in-Aid for JSPS Fellows Grant No. JP18J10205 and JSPS KAKENHI Grant Nos. JP18K03495, JP19H00656, JP19H05826, JP16K17723, and JP19K14630, JST CREST Grant No. JPMJCR1876, the French program Agence nationale de la Recherche DYMESYS (ANR2011-IS04-001-01), and Agence nationale de la Recherche MASH (ANR-12-BS04-0016).

\section{APPENDIX A: SYMMETRIES OF HAMILTONIAN}

In the case where the impurity level is degenerate $\epsilon_{m} \equiv \epsilon_{d}$, the Hamiltonian $\mathcal{H}$ defined in Eq. (1) has the $\mathrm{SU}(N)$ symmetry. This is also owing to the fact that the tunneling matrix element and the Coulomb interaction do not depend on the flavour $m$. It can also be confirmed that $\mathcal{H}$ commutes with each component of the $\mathrm{SU}(N)$ generators:

$$
\boldsymbol{J}^{\mu} \equiv \frac{1}{2} \sum_{m, m^{\prime}=1}^{N}\left[d_{m}^{\dagger} \lambda_{m, m^{\prime}}^{\mu} d_{m^{\prime}}+\sum_{\nu=L, R} \int_{-D}^{D} d \varepsilon c_{\nu, \varepsilon, m}^{\dagger} \lambda_{m, m^{\prime}}^{\mu} c_{\nu, \varepsilon, m}\right]
$$

Here, $\lambda^{\mu}$ for $\mu=1,2, \ldots, N^{2}-1$ are the Gell-mann matrices that satisfy the commutation relations,

$$
\left[\lambda^{i}, \lambda^{j}\right]=2 i \sum_{k=1}^{N^{2}-1} f^{i j k} \lambda^{k},
$$

with $f^{i j k}$ the structure factor. The Hamiltonian $\mathcal{H}$ has also an U(1) tot symmetry and commutes with the total number operator $Q_{\mathrm{tot}}=\sum_{m=1}^{N} Q_{m}$. Here, $Q_{m}$ is the number operator for flavour $m$ :

$$
Q_{m}=\left[n_{d m}+\sum_{\nu=L, R} \int_{-D}^{D} d \varepsilon c_{\nu, \varepsilon, m}^{\dagger} c_{\nu, \varepsilon, m}\right],
$$

for $m=1,2, \ldots, N$. Perturbations that lift the degeneracy of the impurity level lower the symmetry from $\mathrm{SU}(N) \otimes \mathrm{U}(1)_{\text {tot }}$ to $\mathrm{U}(1)_{m=1} \otimes \mathrm{U}(1)_{m=2} \otimes \mathrm{U}(1)_{m=3} \otimes \mathrm{U}(1)_{m=4}$ as $\mathcal{H}$ still commutes with each number operator $Q_{m}$.

For carbon nanotube dots, the two states of $m=2$ and 3 remain degenerated even in magnetic fields when the matching 
condition Eq. (40) holds. In the condition, $\mathcal{H}$ commutes with the SU(2)-pseudospin operator,

$$
\boldsymbol{S}^{\mu} \equiv \frac{1}{2} \sum_{m, m^{\prime}=\Uparrow, \Downarrow}\left[d_{m}^{\dagger} \boldsymbol{\sigma}_{m, m^{\prime}}^{\mu} d_{m^{\prime}}+\sum_{\nu=L, R} \int_{-D}^{D} d \varepsilon c_{\nu, \varepsilon, m}^{\dagger} \boldsymbol{\sigma}_{m, m^{\prime}}^{\mu} c_{\nu, \varepsilon, m}\right]
$$

acting on the two states. Here, $\boldsymbol{\sigma}^{\mu}$ for $\mu=x, y$, and $z$ are the Pauli matrices. The pseudospins $\Uparrow$ and $\Downarrow$ respectively denote the state of $m=2$ and 3. Thus the Hamiltonian has the $\mathrm{U}(1)_{m=1} \otimes[\mathrm{SU}(2) \otimes \mathrm{U}(1)]_{m=2,3} \otimes \mathrm{U}(1)_{m=4}$ symmetries. The SU(2) symmetry plays a central role in the field-induced SU(4) to SU(2) Kondo crossover.

At half-filling point $\epsilon_{d} / U=-3 / 2$, the Hamiltonian $\mathcal{H}$ is invariant under an extended electron-hole transformation:

$$
d_{1}^{\dagger} \Rightarrow h_{4}, \quad d_{2}^{\dagger} \Rightarrow h_{3}, \quad d_{3}^{\dagger} \Rightarrow h_{2}, \quad d_{4}^{\dagger} \Rightarrow h_{1},
$$

and correspondingly, $c_{v, \varepsilon_{m}, m}^{\dagger} \Rightarrow-f_{v,-\varepsilon_{m^{\prime}}, m^{\prime}}$ for $\left(m, m^{\prime}\right)=$ $(1,4),(2,3),(3,2),(4,1)$. Here, $h_{m}$ and $f_{v, \varepsilon_{m^{\prime}}, m^{\prime}}$ annihilate hole in the dot and the conduction bands, respectively.

\section{APPENDIX B: OVERVIEW OF NRG ITERATIONS}

We provide a brief overview of an NRG iteration. The NRG Hamiltonian $\mathcal{H}$ for an Anderson impurity model is a semi-infinite tight-binding chain with the imuprity at a left end. Specifically,

$$
\begin{gathered}
\mathcal{H}_{\mathrm{NRG}}=\mathcal{H}_{d}^{0}+\mathcal{H}_{U}+\mathcal{H}_{\text {chain }}+\mathcal{H}_{T}, \\
\mathcal{H}_{d}^{0}=\sum_{m=1}^{N} \epsilon_{m} d_{m}^{\dagger} d_{m}, \quad \mathcal{H}_{U}=U \sum_{m<m^{\prime}} n_{d m} n_{d m^{\prime}}, \\
\mathcal{H}_{\text {chain }}=\sum_{n=0}^{\infty} \sum_{m=1}^{N} t_{n}\left(f_{n, m}^{\dagger} f_{n+1, m}+\text { H.c. }\right), \\
\mathcal{H}_{T}=V \sum_{m=1}^{N}\left(d_{m}^{\dagger} f_{m, 0}+\text { H.c. }\right) .
\end{gathered}
$$

Here, $\Lambda$ is a logarithmic discretization parameter. A hybridization matrix element $V$ couples the impurity site to a 0 th site of the chain. Similarly, a hopping element $t_{n}$ couples the $n$th site to $(n+1)$ th site. The explicit forms of $V$ and $t_{n}$ are respectively

$$
\begin{gathered}
\frac{V}{D}=\sqrt{\frac{2 \Delta A_{\Lambda}}{\pi D}}, \\
\frac{t_{n}}{D}=\frac{\left(1+\Lambda^{-1}\right)\left(1-\Lambda^{-n-1}\right)}{2\left(1-\Lambda^{-2 n-1}\right)^{1 / 2}\left(1-\Lambda^{-2 n-3}\right)^{1 / 2}} \Lambda^{-n / 2} .
\end{gathered}
$$

Note that the logarithmic discretization corrects the tunneling matrix element $V . A_{\Lambda}$ represents such a correction and can be expressed as

$$
A_{\Lambda}=\frac{1}{2}\left(\frac{1+1 / \Lambda}{1-1 / \Lambda}\right) \ln \Lambda .
$$

This correction $A_{\Lambda}$ approaches 1 in the continuum limit,

$$
\lim _{\Lambda \rightarrow 1} A_{\Lambda}=1 .
$$

The hopping element $t_{n}$ exponentially decays with increasing $n$ and its asymptotic form is

$$
\frac{t_{n}}{D} \sim \frac{1+\Lambda^{-1}}{2} \Lambda^{-n / 2} .
$$

To iteratively diagonalize the Hamiltonian $\mathcal{H}_{\mathrm{NRG}}$, we introduce a Hamitonian $\mathcal{H}^{L}$ which describe the chain ends at an $L$ th site. The explicit form of $\mathcal{H}^{L}$ is

$$
\begin{gathered}
\mathcal{H}^{L}=\Lambda^{(L-1) / 2}\left[\mathcal{H}_{d}^{0}+\mathcal{H}_{U}+\mathcal{H}_{\text {chain }}^{L}+\mathcal{H}_{T}\right], \\
\mathcal{H}_{\text {chain }}^{L}=\sum_{n=0}^{L-1} \sum_{m=1}^{N} t_{n}\left(f_{n, m}^{\dagger} f_{n+1, m}+\text { H.c. }\right) .
\end{gathered}
$$

$\Lambda^{(L-1) / 2}$ is multiplied to the right-hand side of Eq. (B10) to makes the hopping element $\Lambda^{(L-1) / 2} t_{L-1}$ be order of 1 . Eigenvalues of $\mathcal{H}^{L}$ also become order of 1 due to the multiplication. The full Hamiltonian $\mathcal{H}_{\mathrm{NRG}}$ is recovered as the limit of

$$
\mathcal{H}_{\mathrm{NRG}}=\lim _{L \rightarrow \infty} \Lambda^{-(L-1) / 2} \mathcal{H}^{L} .
$$

Here, the prefactor corresponds to the energy scale of lowlying excited states of $\mathcal{H}^{L}$ :

$$
\frac{T_{L}}{D}=\Lambda^{-(L-1) / 2}
$$

To run the iteration, we deduce a recurrence relation for $\mathcal{H}^{L}$ from Eq. (B10),

$$
\mathcal{H}^{L+1}=\Lambda^{1 / 2} \mathcal{H}^{L}+\Lambda^{L / 2} t_{L} \sum_{m=1}^{N}\left(f_{L, m}^{\dagger} f_{L+1, m}+\text { H.c. }\right) \text {. }
$$

Using this relation, we can construct a matrix for $\mathcal{H}^{L+1}$ from eigenvalues and corresponding eigenstates for the Hamiltonian $\mathcal{H}^{L}$. The iterative procedure yields the eigenenergies $E_{L}(\omega)$ at each $L$ th step. we keep only the $N_{K}$ eigenstates with the lowest energies and discard the higher energy states because a dimension of $\mathcal{H}^{L}$ increases with increasing $L$.

Figure 10 plots low-lying eigenenergies of a CNT dot as fucntions of even $L$ with typical parameters: $\Lambda=6.0$, $N_{K}=4100, U /(\pi \Delta)=4.0, \epsilon_{d} / U=-3 / 2$, and $b / T_{K}^{\mathrm{SU}(4)}=$ 1.0. Here, the bare resonance width is $\Delta / D=1 /(100 \pi)$. The SU(4) temperature $T_{K}^{\mathrm{SU}(4)} / \Delta=0.2$ determined at $b=0$ scales the magnetic field $b$. In the value of $b / T_{K}^{\mathrm{SU}(4)}=1.0$, the Fermi-liquid state of the dot is in the middle of the SU(4) to $\mathrm{SU}(2)$ Kondo crossover as shown in Fig. 5(a). Specifically, Fig. 5(a) shows the magnetization $M_{d}$ is still not saturated to 1 at the value of $b$. Figure 10 shows that the energies do not depend on $L$ in the region of $L \gtrsim 20$. At the last iteration $L=$ 30 , the energy scale $T_{L}$ given in Eq. (B13) is much smaller not only than $T_{K}^{\mathrm{SU}(4)}$ but also than the $\mathrm{SU}(2)$ Kondo temperature $T_{K}^{\mathrm{SU}(2)} / \Delta=0.02: T_{L} / \Delta \approx 1.6 \times 10^{-9}, T_{L} / T_{K}^{\mathrm{SU}(4)} \approx$ $8.2 \times 10^{-9}$, and $T_{L} / T_{K}^{S U(2)} \approx 1.0 \times 10^{-7}$. The value of $T_{K}^{\mathrm{SU}(2)}$ is determined at $b \rightarrow \infty$. Therefore the low-lying energy states approach the Fermi-liquid states for $L$ greater than 20 . 


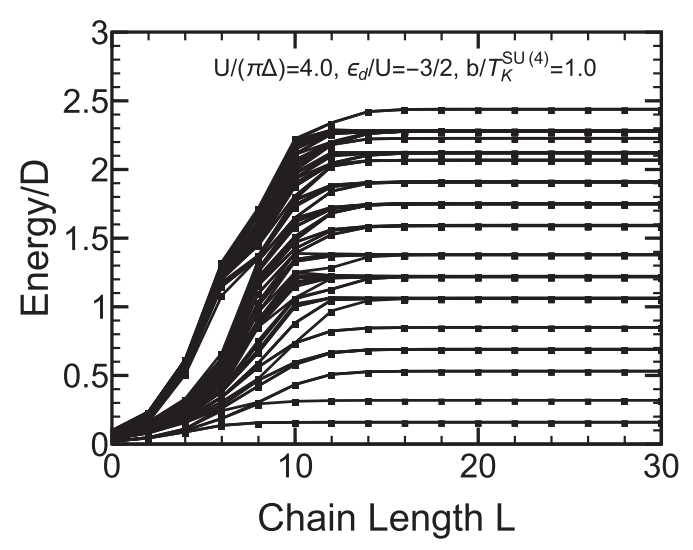

FIG. 10. NRG energy flow of a CNT dot as functions of the even chain length $L$. A CNT dot has $N=4$ internal degrees of freedom. We keep 4100 eigenvalues at each NRG step and plot the lowest 100 values in this figure. The logarithmic discretization parameter is $\Lambda=6$. Values of $U, \epsilon_{d}$, and magnetic field $b$ for the calculation are $U /(\pi \Delta)=4.0, \epsilon_{d} / U=-3 / 2$, and $b / T_{K}^{\mathrm{SU}(4)}=1.0$, respectively. The impurity resonance width is $\Delta / D=1 /(100 \pi)$. The SU(4) temperature determined at $b=0$ is $T_{K}^{\mathrm{SU}(4)} / \Delta=0.2$.

\section{APPENDIX C: CALCULATION METHOD OF FERMI-LIQUID PARAMETERS}

We show how to calculate Fermi-liquid parameters such as phase shifts $\delta_{m}$, renormalization factors $Z_{m}$, and residual interactions $\widetilde{U}_{m, m^{\prime}}$. Let us consider a noninteracting case of the NRG Hamiltonian, i.e., $\mathcal{H}_{\mathrm{NRG}}$ at $U=0$ since a single particle and hole excitation from the ground state determine the phase shifts and the renormalization factors. In such a case, a matrix form of the Hamiltonian $\mathcal{H}^{L}$ is

$$
\mathcal{H}_{U=0}^{L}=\Lambda^{(L-1) / 2}\left[\begin{array}{ccccccc}
\epsilon_{m} & V & & & & & 0 \\
V & 0 & t_{0} & & & \\
0 & t_{0} & 0 & t_{1} & & \\
& & \ddots & \ddots & \ddots & \\
& & & t_{L-2} & 0 & t_{L-1} \\
\mathbf{0} & & & & & t_{L-1} & 0
\end{array}\right] .
$$

The Green's function $G_{d}(\omega)$ at the impurity site $(i=-1)$ is the $(1,1)$ element of the resolvent of this Hamiltonian matrix,

$$
\begin{aligned}
G_{m}(\omega) & \equiv\left(\omega \mathbf{1}-\mathcal{H}_{\mathbf{U}=\mathbf{0}}^{\mathbf{L}}\right)_{1,1}^{-1} \\
& =\frac{1}{\omega-\epsilon_{m} \Lambda^{(L-1) / 2}-V^{2} \Lambda^{(L-1)} g_{00, m}(\omega)} .
\end{aligned}
$$

An eigenvalue $E$ of $\mathcal{H}^{L}$ is a pole of $G_{d}$. Specifically, the value is a solution of the below equation,

$$
E-\epsilon_{m} \Lambda^{(L-1) / 2}-V^{2} \Lambda^{(L-1)} g_{00, m}(E)=0 .
$$

In Eqs. (C2) and (C3), $g_{00, m}(\omega)$ is the Green's function at a site $i=0$ for a chain which starts from the zeroth site and ends at the $L$ th site. A Hamiltonian $\mathcal{H}_{0}^{L}$ describes such a chain. $g_{00, m}(\omega)$ is the $(1,1)$ element of the resolvent of $\mathcal{H}_{0}^{L}$,

$$
g_{00, m}(\omega) \equiv\left(\omega \mathbf{1}-\mathcal{H}_{\mathbf{0}}^{\mathbf{L}}\right)_{1,1}^{-1}
$$

A ratio of the impurity level position $\epsilon_{m}$ to its width $\Delta$ is deduced from Eq. (C3),

$$
\frac{\epsilon_{m}}{\Delta}=\frac{E}{\Delta} \Lambda^{\frac{-(L-1)}{2}}-\frac{2 A_{\Lambda}}{\pi} \lim _{L \rightarrow \infty} \Lambda^{\frac{L-1}{2}} D g_{00, m}(E) .
$$

We here use the relation Eq. (B5) between the hybridization $V$ and $\Delta$.

We henceforth discuss interacting cases, i.e., cases of $U \neq$ 0 , by extending the Eq. (C5) to such cases. Let $E_{p, m}(L)$ denote an excitation energy on adding an electron to the ground state. Similarly, we define $E_{h, m}(L)$ as an excitation energy on adding a hole to the ground state. The NRG iteration procedure calculates $E_{p, m}(L)$ and $E_{h, m}(L)$ as a function of the chain length $L$. We note that $E_{p, m}(L)>0$ and $E_{h, m}(L)<0$. Here we set the energy of the ground state as 0 . Replacing the noninteracting eigenvalue $E$ in Eq. (C5) to the corresponding $E_{p, m}(L)$ or $E_{h, m}(L)$ yields ratios of renormalized level position $\widetilde{\epsilon}_{m}$ to its width $\widetilde{\Delta}_{m}$,

$$
\begin{aligned}
& \frac{\widetilde{\epsilon}_{m, p}}{\widetilde{\Delta}_{m, p}}=\frac{E_{p, m}(L)}{\widetilde{\Delta}_{m, p}} \Lambda^{\frac{-(L-1)}{2}}-\frac{2 A_{\Lambda}}{\pi} \Lambda^{\frac{L-1}{2}} D g_{00, m}\left(E_{p, m}(L)\right) \\
& \frac{\widetilde{\epsilon}_{m, h}}{\widetilde{\Delta}_{m, h}}=\frac{E_{h, m}(L)}{\widetilde{\Delta}_{m, h}} \Lambda^{\frac{-(L-1)}{2}}-\frac{2 A_{\Lambda}}{\pi} \Lambda^{\frac{L-1}{2}} D g_{00, m}\left(E_{h, m}(L)\right)
\end{aligned}
$$

Here, $\widetilde{\epsilon}_{m, p}$ and $\widetilde{\epsilon}_{m, h}$ are the renormalized resonance level positions calculated from $E_{p, m}$ and $E_{h, m}$, respectively. Similarly, $\widetilde{\Delta}_{m, p}$ and $\widetilde{\Delta}_{m, h}$ are the renormalized line widths from $E_{p, m}$ and $E_{h, m}$. The first terms in the right-hand side of the equations can be neglected in the limit of $L \rightarrow \infty$ because $E_{p, m}(L)$ and $E_{h, m}(L)$ are of order 1 . Thus the ratios become

$$
\frac{\widetilde{\epsilon}_{p(h), m}}{\widetilde{\Delta}_{p(h), m}}=-\frac{2 A_{\Lambda}}{\pi} \lim _{L \rightarrow \infty} \Lambda^{\frac{L-1}{2}} D g_{00, m}\left(E_{p(h), m}(L)\right) .
$$

We obtain occupation numbers $\left\langle n_{d m, p}\right\rangle$ and $\left\langle n_{d m, h}\right\rangle$ by substituting the ratios into the Friedel sum rule given by

$$
\left\langle n_{m, p(h)}\right\rangle=\frac{1}{2}-\tan ^{-1}\left(\frac{\widetilde{\epsilon}_{p(h), m}}{\widetilde{\Delta}_{p(h), m}}\right) .
$$

Furthermore, subtracting Eq. (C7) from Eq. (C6) gives the renormalized level width $\widetilde{\Delta}_{m}$,

$$
\widetilde{\Delta}_{m}=\frac{\pi}{2 A_{\Lambda}} \frac{E_{p, m}(L)-E_{h, m}(L)}{\Lambda^{(L-1)} D g_{00, m}\left(E_{p, m}(L)\right)-\Lambda^{(L-1)} D g_{00, m}\left(E_{h, m}(L)\right)} .
$$

Figure 11(a) plots the occupation numbers of the CNT dot as functions of the NRG chain length $L$. The parameters are $U /(\pi \Delta)=4.0, \epsilon_{d} / U=-3 / 2$, and $b / T_{K}^{\mathrm{SU}(4)}=1.0$ for the plots. The SU(4) Kondo temperature is $T_{K}^{\mathrm{SU}(4)} / \Delta=$ 0.2 for the values of $U$ and $\epsilon_{d}$. Figure 11(a) clearly shows that $\left\langle n_{1, p}\right\rangle$ from the particle excitation and $\left\langle n_{1, h}\right\rangle$ from the hole excitation converge to a same value $\left\langle n_{1}\right\rangle=0.84$ with increasing $L$. $\left\langle n_{2, p}\right\rangle$ and $\left\langle n_{2, h}\right\rangle$ also converge to the half-filled value $\left\langle n_{2}\right\rangle=0.5$. Since the extended electron-hole symmetry described by Eq. (A5) imposes relations between the occupation numbers: $\left\langle n_{4, p}\right\rangle=1-\left\langle n_{1, h}\right\rangle$ and $\left\langle n_{4, h}\right\rangle=1-\left\langle n_{1, p}\right\rangle$, $\left\langle n_{4, p}\right\rangle$ and $\left\langle n_{4, h}\right\rangle$ converge to a value $1-\left\langle n_{1}\right\rangle=0.16$. The values of $\left\langle n_{d, 1}\right\rangle$ and $\left\langle n_{d, 4}\right\rangle$ give a value of the magnetization 

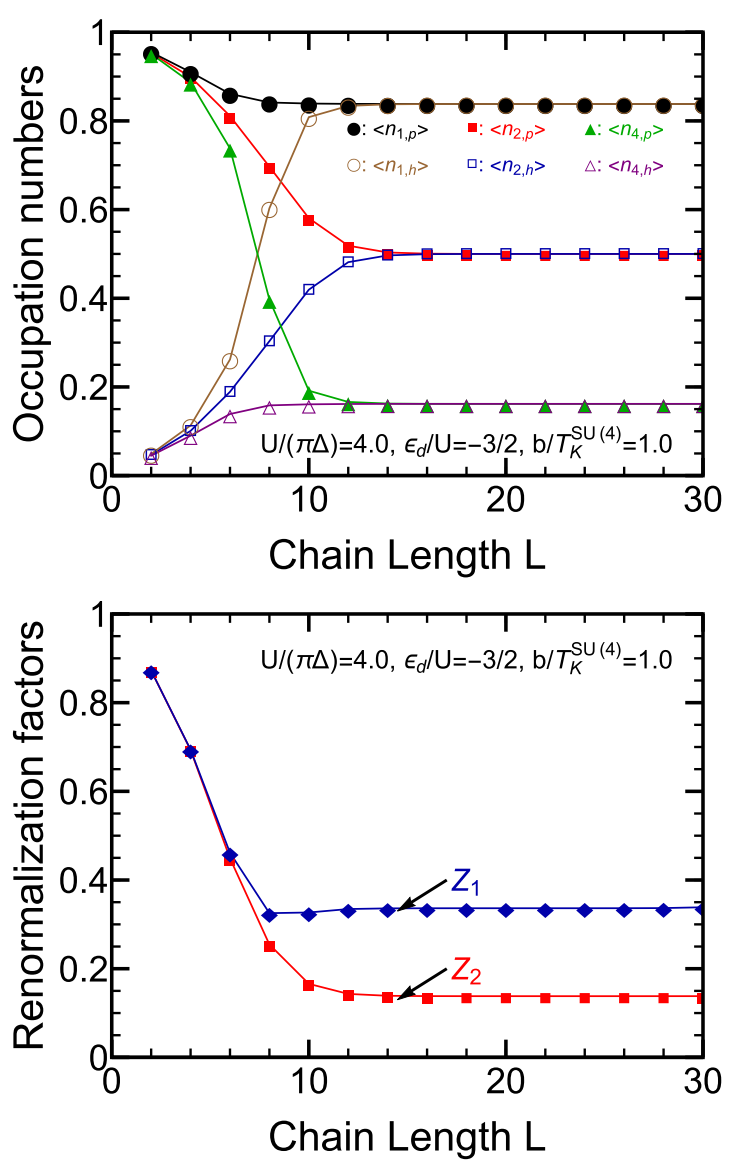

FIG. 11. (a) shows flows of occupation numbers: $\left\langle n_{1, p}\right\rangle,\left\langle n_{1, h}\right\rangle$, $\left\langle n_{2, p}\right\rangle,\left\langle n_{2, h}\right\rangle,\left\langle n_{4, p}\right\rangle$, and $\left\langle n_{4, h}\right\rangle$. (b) shows those of renormalization factors: $Z_{1}$ and $Z_{2}$. The $x$ axis is the NRG chain length $L$ in each figure. In (a), Following six symbols, $\bullet, \circ, \mathbf{\square}, \square, \boldsymbol{\Delta}$, and $\triangle$ respectively denote the values of $\left\langle n_{1, p}\right\rangle,\left\langle n_{1, h}\right\rangle,\left\langle n_{2, p}\right\rangle,\left\langle n_{2, h}\right\rangle,\left\langle n_{4, p}\right\rangle$, and $\left\langle n_{4, h}\right\rangle$. In (b), and respectively denote the values of $Z_{1}$ and $Z_{2}$. The parameters are $U /(\pi \Delta)=4.0, \epsilon_{d} / U=-3 / 2$, and $b / T_{K}^{\mathrm{SU}(4)}=1.0$ for each figure. These parameters are the same as those for Fig. 10. The SU(4) Kondo temperature is $T_{K}^{\mathrm{SU}(4)} / \Delta=0.2$ for the values of $U$ and $\epsilon_{d}$. Eq. (41) describes the magnetic filed $b$ dependence of the four dot levels $\epsilon_{1}, \epsilon_{2}, \epsilon_{3}$, and $\epsilon_{4}$.

$M_{d}=\left\langle n_{d, 1}\right\rangle-\left\langle n_{d, 4}\right\rangle=0.67$. Figure 5(a) shows this value of $M_{d}$ at the point of $b / T_{K}^{\mathrm{SU}(4)}=1.0$.

Figure 11(b) plots the chain length $L$ dependence of renormalization factors $Z_{m}$, which are ratios of $\widetilde{\Delta}_{m}$ to $\Delta$, i.e., $Z_{m}=$ $\widetilde{\Delta}_{m} / \Delta$. We note that the electron-hole symmetry also makes two of the four factors independent: $Z_{1}=Z_{4}$ and $Z_{2}=Z_{3}$ This figure shows that values of $Z_{1}$ and $Z_{2}$ respectively converge to $Z_{1}=0.34$ and $Z_{2}=0.14$. Since the value of $Z_{2}$ is about ten times as large as the $S U(2)$ limit value 0.026, the Fermiliquid state at $b / T_{K}^{\mathrm{SU}(4)}=1.0$ is in the middle of the crossover between the SU(4) and SU(2) Kondo state.

The NRG iteration also yields higher excitation energies from the ground state. $E_{k, p, m}$ and $E_{k, h, m}$ respectively represent such electron and hole excitation energies. We note that $E_{k, p, m}>0$ and $E_{k, h, m}<0$. They describe a free quasiparticle Hamiltonian,

$$
\mathcal{H}^{0}=\sum_{m=1}^{N} \sum_{k}\left(E_{k, p, m} p_{k, m}^{\dagger} p_{k, m}-E_{k, h, m} h_{k, m}^{\dagger} h_{k, m}\right) .
$$

Here, operators $p_{k, m}^{\dagger}$ and $h_{k, m}^{\dagger}$ respectively create the quasiparticle with $E_{k, p, m}$ and the quasihole with $E_{k, h, m}$. Correspondingly, $p_{k, m}$ and $h_{k, m}$ annihilate the quasiparticle and quasihole. The ground state $|0\rangle$ is defined such that $p_{k, m}|0\rangle=0$ and $h_{k, m}|0\rangle=0$. A linear combination of $p_{k, m}^{\dagger}$ and $h_{k, m}$ expresses the operator $d_{m}^{\dagger}$ which creates the impurity electron,

$$
d_{m}^{\dagger}=\sum_{k}\left(p_{k, m}^{\dagger} \phi_{k, p, m}(-1)+h_{k, m} \phi_{k, h, m}(-1)\right) \text {. }
$$

To calculate Wilson ratios $R_{m, m^{\prime}}$ defined in Eq. (18), we consider a residual interaction term,

$$
\mathcal{H}_{L}^{U}=\Lambda^{(L-1) / 2} \sum_{m<m^{\prime}} \tilde{U}_{m, m^{\prime}} \mathrm{N}\left[n_{d m} n_{d m^{\prime}}\right] .
$$

Here, $\widetilde{U}_{m, m^{\prime}}$ is the strength of the residual interaction between the quasiparticles. $\mathrm{N}$ is the normal ordering operator. We examine how the interaction affects the low-lying many-particle states to a first order perturbation in $\widetilde{U}_{m, m^{\prime}}$. This order calculation accurately describes the effects of the interaction on the low-energy states because the term vanishes in the limit of $L \rightarrow \infty$. For the explicit calculation, we introduce a twoparticle excited state from the ground state:

$$
|A\rangle=p_{1, m}^{\dagger} p_{1, m^{\prime}}^{\dagger}|0\rangle,
$$

where $m \neq m^{\prime}$. The NRG iteration calculates a corresponding eigenvalue $E_{p p}(L)$. The first order calculation of $\widetilde{U}_{m, m^{\prime}}$ also gives $E_{p p}$,

$$
\begin{aligned}
E_{p p}(L) & =\left\langle A\left|\mathcal{H}^{0}+\mathcal{H}_{L}^{U}\right| A\right\rangle \\
& =2 E_{p, m}(L)+\widetilde{U}_{m, m^{\prime}} \Lambda^{-\frac{L-1}{2}}\left|\beta_{1, p, m}\right|^{2}\left|\beta_{1, p, m^{\prime}}\right|^{2}
\end{aligned}
$$

In this equation, $\left|\beta_{1, p, m}\right|^{2}$ is given by

$$
\left|\beta_{1, p, m}\right|^{2}=\frac{\Lambda^{(L-1) / 2}}{1-\left.\widetilde{V}_{m}^{2} \Lambda^{(L-1)} \frac{d g_{00, m}(E)}{d E}\right|_{E=E_{1, p, m}}},
$$

where $\widetilde{V}_{m}^{2}=2 D \widetilde{\Delta}_{m} A_{\Lambda} / \pi$. The $L$ dependent $\widetilde{U}_{m, m^{\prime}}(L)$ is deducible from Eq. (C15) since the NRG iteration procedure determines $E_{p p}(L)$ in the left hand of the equation. We can alternatively consider two hole excitations to calculate $\widetilde{U}_{m, m^{\prime}}$. Considering particle-hole excitations and hole-particle excitations also yields $\widetilde{U}_{m, m^{\prime}}$. Here, the word "particle-hole excitations" denote one particle excitation with $m$ flavour and one hole excitation with $m^{\prime}$ flavour. The word "hole-particle excitations" similarly denote one hole and one particle excitation with $m$ and $m^{\prime}$. Thus $\widetilde{U}_{m, m^{\prime}}$ is calculable by four different ways. $\widetilde{U}_{m, m^{\prime}}^{p p}(L), \widetilde{U}_{m, m^{\prime}}^{h h}(L), \widetilde{U}_{m, m^{\prime}}^{p h}(L)$, and $\widetilde{U}_{m, m^{\prime}}^{h p}(L)$ denote the residual interaction deduced from the two-particle, two-hole, particle-hole and hole-particle excitation energies, respectively.

Figures 12(a)-12(c) respectively plot $L$ dependence of $\widetilde{U}_{2,3}, \widetilde{U}_{1,2}$, and $\widetilde{U}_{1,4}$ which are calculated by the four ways. The three figures show that $\widetilde{U}_{m, m^{\prime}}^{p p}, \widetilde{U}_{m, m^{\prime}}^{h h}, \widetilde{U}_{m, m^{\prime}}^{p h}$, and $\widetilde{U}_{m, m^{\prime}}^{h p}$ converge a same value $\widetilde{U}_{m, m^{\prime}}$ for each $\left(m, m^{\prime}\right)$. Each inset clearly shows such convergence of the four values. The converged values are $\widetilde{U}_{2,3} /(\pi \Delta)=0.13, \widetilde{U}_{1,2} /(\pi \Delta)=0.05$, and $\widetilde{U}_{1,4} /(\pi \Delta)=$ 0.35 . 

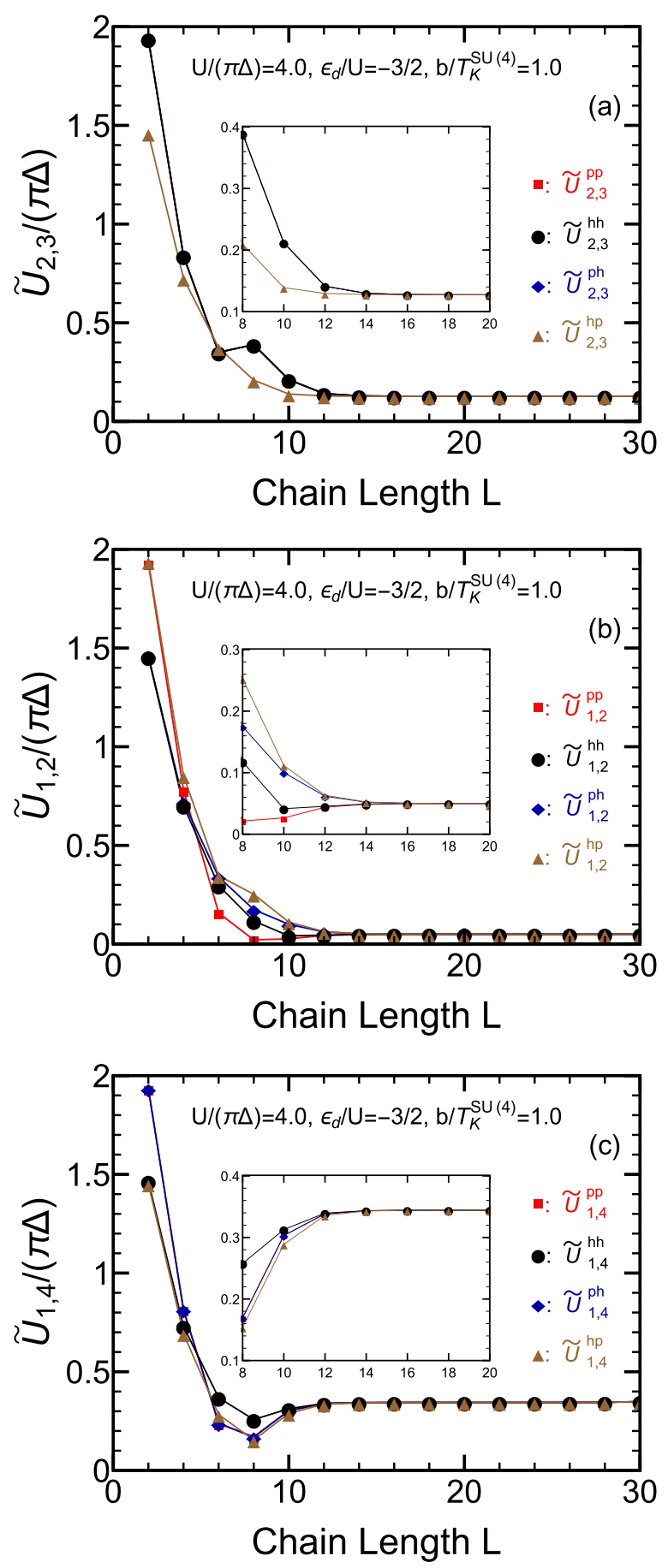

FIG. 12. Residual interactions $\widetilde{U}_{m, m^{\prime}}$ are plotted as functions of the chain length $L$. (a), (b), and (c) respectively show $\left(m, m^{\prime}\right)=$ $(2,3),(1,2)$, and $(1,4)$ element of $\widetilde{U}_{m, m^{\prime}}$. The particle-hole symmetry given by Eq. (A5) imposes following relations to the residual interactions: $\widetilde{U}_{1,2}=\widetilde{U}_{1,3}=\widetilde{U}_{2,4}=\widetilde{U}_{3,4}$. The parameters are $U /(\pi \Delta)=4.0$, $\epsilon_{d} / U=-3 / 2$, and $b / T_{K}^{\mathrm{SU}(4)}=1.0$ for every three figure. The SU(4) Kondo temperature is $T_{K}^{\mathrm{SU}(4)} / \Delta=0.2$ for the values of $U$ and $\epsilon_{d}$. Following four symbols: $\mathbf{\square}, \bullet, \diamond$, and $\boldsymbol{\Delta}$ respectively represent $\widetilde{U}_{m, m^{\prime}}^{p p}$, $\widetilde{U}_{m, m^{\prime}}^{h h}, \widetilde{U}_{m, m^{\prime}}^{p h}$, and $\widetilde{U}_{m, m^{\prime}}^{h p}$ in each figure. The insets show an enlarged view in the range of $8 \leqslant L \leqslant 20$.

The Wilson ratios $R_{m, m^{\prime}}$ are deducible from the obtained values of $\delta_{m}=\left\langle n_{d m}\right\rangle / \pi, Z_{m}$, and $\widetilde{U}_{m, m^{\prime}}$ using Eqs. (54)-(56). Figure 13 plots $R_{2,3}-1, R_{1,4}-1$, and $R_{1,2}-1$ as func-

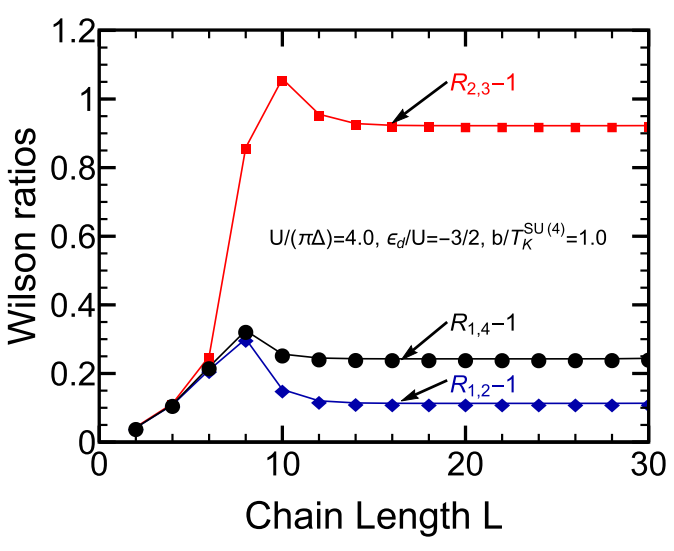

FIG. 13. Wilson ratios $R_{2,3}-1, R_{1,4}-1$, and $R_{1,2}-1$ are plotted as functions of the chain length $L$ for the parameters: $U /(\pi \Delta)=$ $4.0, \epsilon_{d} / U=-3 / 2$, and $b / T_{K}^{\mathrm{SU}(4)}=1.0$. Each three symbol, i.e., $\mathbf{\square}$, $\bullet$, and represents values of $R_{2,3}-1, R_{1,4}-1$, and $R_{1,2}-1$, respectively. The SU(4) Kondo temperature is $T_{K}^{\mathrm{SU}(4)} / \Delta=0.2$ for the values of $U$ and $\epsilon_{d}$.

tions of $L$. The four values of $\widetilde{U}_{m, m^{\prime}}^{p p}, \widetilde{U}_{m, m^{\prime}}^{h h}, \widetilde{U}_{m, m^{\prime}}^{p h}$, and $\widetilde{U}_{m, m^{\prime}}^{h p}$ are averaged to calculate the ratios $R_{m, m^{\prime}}-1$. Each of the three ratios converges for $L \gtrsim 20$. The converged values are $R_{2,3}-1=0.92, R_{1,2}-1=0.11$, and $R_{1,4}-1=0.24 . R_{2,3}$ becomes larger than the other two ratios because the corresponding impurity levels of $R_{2,3}$ remain the Fermi level.

\section{APPENDIX D: FERMI-LIQUID PARAMETERS FOR SINGLE ORBITAL ANDERSON IMPURITY}

We briefly discuss how the Fermi liquid state of the single Anderson impurity evolves with increasing magnetic field. The field dependence of the Fermi liquid parameters have been discussed also in Refs. [65,66]. Figures 14(a) and 14(b) plot NRG results of the Fermi liquid parameters and the residual interaction, respectively. In the NRG calculations, the spin-dependent impurity level is chosen such that $\varepsilon_{d, \sigma}=$ $\varepsilon_{d}+\operatorname{sgn}(\sigma) b$, and the center of the impurity level is locked at the half-filling, $\varepsilon_{d}=-U / 2$. Figure 14(a) shows that the transmission probability $T(0)=\sin ^{2} \delta$ decreases as soon as magnetic fields become comparable with the Kondo temperature $T_{K}^{\mathrm{SU}(2)}$, and correspondingly, the induced magnetization is rapidly saturated to 1 , i.e., $M_{d} \rightarrow 1$. In contrast, $Z$ and $R-1$ vary more slowly than $T(0)$ and $M_{d}$ with the scales of $U$. The inset of Fig. 14(a) shows that $\widetilde{\Delta}$ and $R-1$ are still renormalized for small magnetic fields $b \lesssim T_{K}^{\mathrm{SU}(2)}$. For large magnetic fields $b \gg T_{K}^{\mathrm{SU}(2)}$, these parameters approach the noninteracting values, $Z \rightarrow 1$ and $R-1 \rightarrow 1$.

The residual interaction plotted in Fig. 14(b) also varies from a zero field value $4 T_{K}^{\mathrm{SU}(2)}=0.23 \pi \Delta$ with increasing $b$. For small magnetic fields $b \lesssim 0.2 U, \widetilde{U}$ is enhanced and its value becomes larger than the bare Coulomb interaction $U$. As magnetic fields further increase, it decreases from the enhanced value to the bare value $U$.

\section{APPENDIX E: NRG FOR DYNAMICAL CORRELATIONS}

\section{Spectral function and self-energy}

In this work, the spectral function $A_{m}(\omega)$ has been calculated using the "complete Fock-space basis set," developed by 
Andes et al. [24,25] and by Weichselbaum and von Delft [26]. In this approach, contributions of the high energy states which are discarded at the NRG steps can be recovered to form a complete basis for the Wilson's NRG chain, by carrying out the backward iteration. The merit of this approach is that the sum rule for the spectral weights can be fulfilled.

We have also employed the method due to Bulla, Hewson, and Pruschke [62]: we have calculated not only $G_{m}(\omega)$ but also the higher-order Green's function $F_{m}(\omega)$

$$
\begin{aligned}
F_{m}(\omega)=- & i \int_{0}^{\infty} d t e^{i\left(\omega+i 0^{+}\right) t} \\
& \times \sum_{m^{\prime}(\neq m)}\left\langle\left\{n_{d m^{\prime}}(t) d_{m}(t), d_{m}^{\dagger}(0)\right\}\right\rangle .
\end{aligned}
$$

Then, the self-energy can be determined directly through the relation $\Sigma_{m}(\omega)=U F_{m}(\omega) / G_{m}(\omega)$. The final form of the Green's function has been obtained from $\Sigma_{m}(\omega)$ and the noninteracting Green's function $G_{m}^{0}(\omega)$ using the Dyson equation given in Eq. (7). The merit to treat the self-energy as an input is that the fully analytic expression which is not affected by the logarithmic discretization can be used for $G_{m}^{0}(\omega)$.

\section{The zaveraging}

The size of the Hilbert space to be diagonalized at each NRG step increases as the number of conduction electron channels increases. To ensure the accuracy of the NRG calculation, a large $\Lambda$ is used for quantum impurities with a number of internal degrees of freedom.

Oliveira and Oliveira found that thermodynamic averages which are calculated for large $\Lambda$ show an artificial oscillation at low temperatures $[59,61]$, and they proposed the $z$ averaging for removing such an artificial oscillation. The parameter $z$ slides a set of discretization points from that of the standard Wilson chain [22],

$$
\pm \Lambda^{-n} \rightarrow \pm \Lambda^{-(n+1-z)}, \quad n=0,1,2, \ldots,
$$

with $0 \leqslant z \leqslant 1$. For $z=1$, it coincides with the standard Wilson chain. The discretized conduction band can be transformed into a $z$ dependent Wilson chain

$$
\mathcal{H}_{c} \Rightarrow \sum_{n=0}^{\infty} \sum_{m=1}^{4} t_{n}(z)\left(f_{n, m}^{\dagger} f_{n+1, m}+f_{n+1, m}^{\dagger} f_{n, m}\right) .
$$

The hopping matrix element $t_{n}(z)$ that can be determined using the Hauseholder algorithm summarized in Refs. [59,61]. We have carried out NRG calculations for some fixed values of $z$, and calculate expectation values using the obtained eigenstates. Then, an average is taken over " $z$ " for two different values $z=0.5$ and 1 , which is enough to eliminate the artificial oscillations in our case.

\section{Logarithmic-Gaussian function to broaden discrete spectral function}

We have calculated the spectral function $A_{m}$ using the Lehman representation given in Eq. (F1). The resulting function is a set of discrete $\delta$ functions at frequencies $\omega_{n}$. Replacing the $\delta$ function to the logarithmic-Gaussian function makes $A_{m}$ continuous since the NRG energy scale $T_{L}$ given
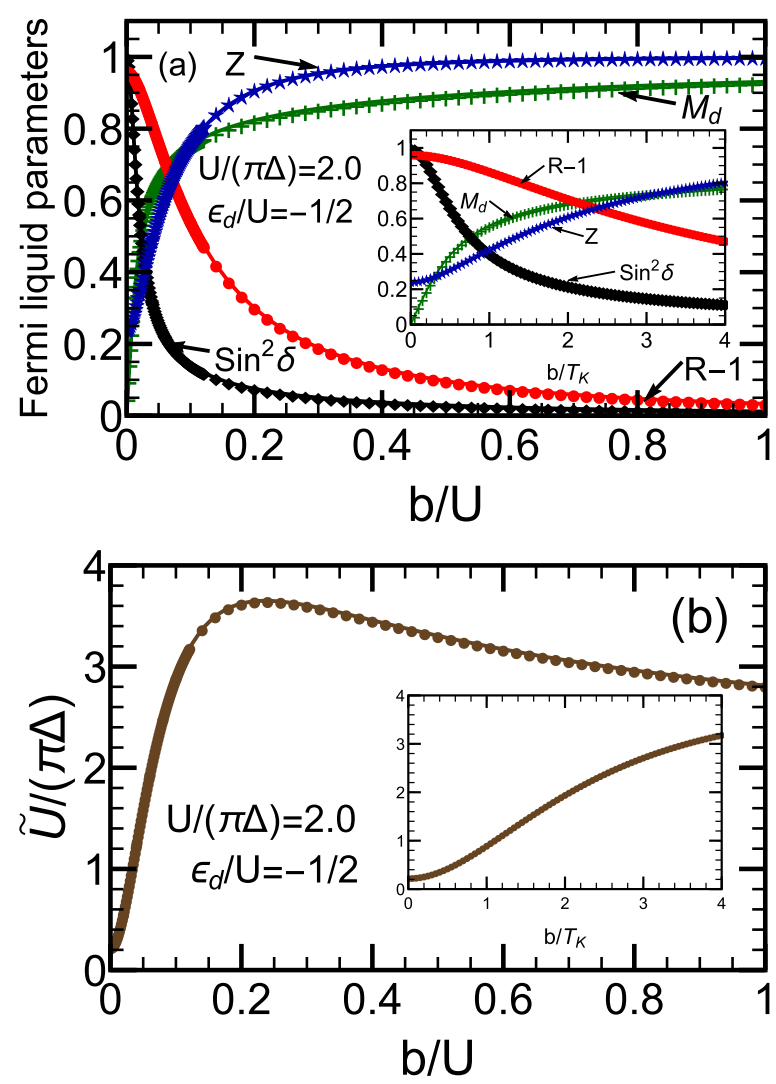

FIG. 14. (a) Magnetic field dependence of Fermi liquid parameters for single orbital Anderson impurity at the electron-hole symmetric point $\varepsilon_{d} / U=-1 / 2$ for $U /(\pi \Delta)=2.0$. At this point, a total occupation number is locked at one, i.e., $\left\langle n_{d \downarrow}\right\rangle+\left\langle n_{d \uparrow}\right\rangle=1$ at arbitrary magnetic fields, and thus $\left\langle n_{d \sigma}\right\rangle$ can be expressed in terms of the magnetization $M_{d} \equiv\left\langle n_{d \uparrow}\right\rangle-\left\langle n_{d \downarrow}\right\rangle$ as follows: $\left\langle n_{d \sigma}\right\rangle=$ $\left(1+\operatorname{sgn}(\sigma) M_{d}\right) / 2$. Furthermore, $\sin ^{2} \delta_{\uparrow}=\sin ^{2} \delta_{\downarrow}$ and $Z_{\uparrow}=Z_{\downarrow}$. (b) Residual interaction $\widetilde{U}$ as a function of $b$. The $x$ axis is scaled by the Coulomb interaction $U$. Each inset shows an enlarged view of the region around $b=0$. In the insets, the axis is scaled by the Kondo temperature $T_{K}^{\mathrm{SU}(2)}=0.19 \Delta=(0.03 U)$.

in Eq. (B13) exponentially falls of with increasing the chain length $L$. The function to broaden a particle excitation peak at $\omega_{n}>0$ is

$$
X(\omega)=\frac{\mathrm{e}^{-b^{2} / 4}}{b \omega_{n} \sqrt{\pi}} \exp \left\{-\left(\frac{\ln \left(\omega / \omega_{n}\right)}{b}\right)^{2}\right\},
$$

which is defined for $\omega>0$. Correspondingly, a hole excitation peak at $\omega_{n}<0$ is broadened by

$$
Y(\omega)=\frac{\mathrm{e}^{-b^{2} / 4}}{b\left|\omega_{n}\right| \sqrt{\pi}} \exp \left\{-\left(\frac{\ln \left|\omega / \omega_{n}\right|}{b}\right)^{2}\right\},
$$

for $\omega<0$. We set a broadening parameter $b=1.1$ for the spectral functions plotted in Figs. 8 and 9. Note that the spectral weight is conserved because the broadening functions are normalized to 1 .

\section{APPENDIX F: SPECTRAL FUNCTION IN ATOMIC LIMIT}

We consider the atomic limit in order to show how the spectral weight of the impurity states evolves as magnetic 
increases at high energies in the case that the Zeemann splittings of the impurity levels are given by Eq. (41).

At zero temperature, the flavour $m$-resolved single-particle spectral function can be written in the Lehmann representation as

$$
\begin{aligned}
A_{m}(\omega)= & \frac{1}{M} \sum_{i=1}^{M} \sum_{n}\left[\mid\left\langle\left. n\left|d_{m}^{\dagger}\right| \Psi_{\mathrm{GS}, i}\right|^{2} \delta\left(\omega-\left(E_{n}-E_{\mathrm{GS}}\right)\right)\right.\right. \\
& \left.+\left|\left\langle n\left|d_{m}\right| \Psi_{\mathrm{GS}, i}\right\rangle\right|^{2} \delta\left(\omega+\left(E_{n}-E_{\mathrm{GS}}\right)\right)\right] . \quad(\mathrm{F} 1)
\end{aligned}
$$

Here, $|n\rangle$ and $E_{n}$ are the eigenstate and eigenenergy of Hamiltonian $\mathcal{H}$, respectively. The ground state $\left|\Psi_{\mathrm{GS}, i}\right\rangle$ with the energy $E_{\mathrm{GS}}$ can generally be degenerate, and the summation over $i$ represents an average over $M$-fold degenerate states.

In the atomic limit, the CNT dot whose eigenenergies are defined is Eq. (41) is disconnected from the leads $\left(v_{v}=0\right.$ for $v=L, R$ ), and there remains twofold degeneracy for the ground state at half-filling $\varepsilon_{d}=-3 U / 2$,

$$
\begin{gathered}
\left|\Psi_{\mathrm{GS}, 2}\right\rangle=d_{1}^{\dagger} d_{2}^{\dagger}|0\rangle, \\
\left|\Psi_{\mathrm{GS}, 3}\right\rangle=d_{1}^{\dagger} d_{3}^{\dagger}|0\rangle .
\end{gathered}
$$

Either of the two one-particle states, $m=2$ or 3 situated on the Fermi level, is occupied, and the lowest one-particle level with the energy $\varepsilon_{1}$ is occupied while the highest one with $\varepsilon_{4}$ is empty. Therefore the ground energy for these two-electron states becomes

$$
E_{\mathrm{GS}}=\varepsilon_{1}+\varepsilon_{2}+U=2 \varepsilon_{d}-2 b+U .
$$

We next consider a single-particle excitation to add an electron into the level of $m=4$, and a single-hole excitation to remove the electron occupying the $m=1$ level,

$$
\begin{gathered}
\left|\Psi_{p 4}\right\rangle=d_{4}^{\dagger}\left|\Psi_{\mathrm{GS}, i}\right\rangle, \quad E_{p 4}=3 \varepsilon_{d}+3 U, \\
\left|\Psi_{h 1}\right\rangle=d_{1}\left|\Psi_{\mathrm{GS}, i}\right\rangle, \quad E_{h 1}=\varepsilon_{d} .
\end{gathered}
$$

The excitation energies from the ground state $i=2$ and 3 to these two states are given by

$$
\begin{aligned}
& E_{p 4}-E_{\mathrm{GS}}=\varepsilon_{d}+2 b+2 U=2 b+\frac{U}{2}, \\
& E_{\mathrm{GS}}-E_{h 1}=\varepsilon_{d}-2 b+U=-2 b-\frac{U}{2} .
\end{aligned}
$$

Therefore the spectral weights of these processes are given by

$$
\begin{aligned}
& A_{4}(\omega)=\delta\left(\omega-\left(2 b+\frac{U}{2}\right)\right), \\
& A_{1}(\omega)=\delta\left(\omega+\left(2 b+\frac{U}{2}\right)\right) .
\end{aligned}
$$

These weights shift towards high-energy region from the usual atomic limit position $\pm U / 2$. We have observed the corresponding shifts of the spectral weight in the NRG results shown in Fig. 8(c) and 9(c) although these atomic peaks merge with the resonance peak which also moves away from the Fermi level as $b$ increases.

The other single electron (hole) excitation from the ground state $\left|\Psi_{\mathrm{GS}, 2}\right\rangle$ corresponds to an addition of an electron to the level $m=3$ (annihilation of an electron from $m=2$ ). The similar excitations also occur from $\left|\Psi_{\mathrm{GS}, 3}\right\rangle$. The peaks corresponding to these excitations appear at $\omega= \pm U / 2$ in the spectral functions for $m=2$ and 3 ,

$$
A_{2}(\omega)=A_{3}(\omega)=\frac{1}{2} \delta\left(\omega-\frac{U}{2}\right)+\frac{1}{2} \delta\left(\omega+\frac{U}{2}\right)
$$

These two peaks are equivalent to the Hubbard peaks for the $\mathrm{SU}(2)$ symmetric case.
[1] J. Kondo, Prog. Theor. Phys. 32, 37 (1964).

[2] A. C. Hewson, The Kondo Problem to Heavy Fermions (Cambridge University Press, Cambridge, UK, 1993).

[3] L. I. Glazman and M. E. Raŭkh, JETP Lett. 47, 452 (1988).

[4] T. K. Ng and P. A. Lee, Phys. Rev. Lett. 61, 1768 (1988).

[5] D. Goldhaber-Gordon, J. Göres, M. A. Kastner, H. Shtrikman, D. Mahalu, and U. Meirav, Phys. Rev. Lett. 81, 5225 (1998).

[6] W. Van der Wiel, S. De Franceschi, T. Fujisawa, J. Elzerman, S. Tarucha, and L. Kouwenhoven, Science 289, 2105 (2000).

[7] E. A. Laird, F. Kuemmeth, G. A. Steele, K. Grove-Rasmussen, J. Nygård, K. Flensberg, and L. P. Kouwenhoven, Rev. Mod. Phys. 87, 703 (2015).

[8] S. Sasaki, S. De Franceschi, J. M. Elzerman, W. G. van der Wiel, M. Eto, S. Tarucha, and L. P. Kouwenhoven, Nature (London) 405, 764 (2000).

[9] M.-S. Choi, R. López, and R. Aguado, Phys. Rev. Lett. 95, 067204 (2005).

[10] W. Izumida, O. Sakai, and Y. Shimizu, J. Phys. Soc. Jpn. 67, 2444 (1998).
[11] L. Borda, G. Zaránd, W. Hofstetter, B. I. Halperin, and J. von Delft, Phys. Rev. Lett. 90, 026602 (2003).

[12] F. B. Anders, D. E. Logan, M. R. Galpin, and G. Finkelstein, Phys. Rev. Lett. 100, 086809 (2008).

[13] I. Weymann, R. Chirla, P. Trocha, and C. P. Moca, Phys. Rev. B 97, 085404 (2018).

[14] Y. Nishikawa, A. C. Hewson, D. J. G. Crow, and J. Bauer, Phys. Rev. B 88, 245130 (2013).

[15] A. Makarovski, A. Zhukov, J. Liu, and G. Finkelstein, Phys. Rev. B 75, 241407(R) (2007).

[16] A. J. Keller, S. Amasha, I. Weymann, C. P. Moca, I. G. Rau, J. A. Katine, H. Shtrikman, G. Zaránd, and D. GoldhaberGordon, Nat. Phys. 10, 145 (2014).

[17] P. Jarillo-Herrero, J. Kong, H. S. J. van der Zant, C. Dekker, L. P. Kouwenhoven, and S. De Franceschi, Nature (London) 434, 484 (2005).

[18] K. J. G. Götz, D. R. Schmid, F. J. Schupp, P. L. Stiller, C. Strunk, and A. K. Hüttel, Phys. Rev. Lett. 120, 246802 (2018). 
[19] M. Ferrier, T. Arakawa, T. Hata, R. Fujiwara, R. Delagrange, R. Weil, R. Deblock, R. Sakano, A. Oguri, and K. Kobayashi, Nat. Phys. 12, 230 (2016).

[20] T. Hata, R. Delagrange, T. Arakawa, S. Lee, R. Deblock, H. Bouchiat, K. Kobayashi, and M. Ferrier, Phys. Rev. Lett. 121, 247703 (2018).

[21] K. G. Wilson, Rev. Mod. Phys. 47, 773 (1975).

[22] H. R. Krishna-murthy, J. W. Wilkins, and K. G. Wilson, Phys. Rev. B 21, 1003 (1980).

[23] H. R. Krishna-murthy, J. W. Wilkins, and K. G. Wilson, Phys. Rev. B 21, 1044 (1980).

[24] F. B. Anders and A. Schiller, Phys. Rev. Lett. 95, 196801 (2005).

[25] R. Peters, T. Pruschke, and F. B. Anders, Phys. Rev. B 74, 245114 (2006).

[26] A. Weichselbaum and J. von Delft, Phys. Rev. Lett. 99, 076402 (2007).

[27] M. R. Galpin, F. W. Jayatilaka, D. E. Logan, and F. B. Anders, Phys. Rev. B 81, 075437 (2010).

[28] D. Mantelli, C. P. Moca, G. Zaránd, and M. Grifoni, Physica E 77, 180 (2016).

[29] P. Roura-Bas, L. Tosi, A. A. Aligia, and K. Hallberg, Phys. Rev. B 84, 073406 (2011).

[30] C. Mora, P. Vitushinsky, X. Leyronas, A. A. Clerk, and K. Le Hur, Phys. Rev. B 80, 155322 (2009).

[31] C. Mora, C. P. Moca, J. von Delft, and G. Zaránd, Phys. Rev. B 92, 075120 (2015).

[32] M. Filippone, C. P. Moca, A. Weichselbaum, J. von Delft, and C. Mora, Phys. Rev. B 98, 075404 (2018).

[33] A. Oguri and A. C. Hewson, Phys. Rev. B 97, 035435 (2018).

[34] Y. Teratani, R. Sakano, and A. Oguri, arXiv:2001.08348.

[35] M. Ferrier, T. Arakawa, T. Hata, R. Fujiwara, R. Delagrange, R. Deblock, Y. Teratani, R. Sakano, A. Oguri, and K. Kobayashi, Phys. Rev. Lett. 118, 196803 (2017).

[36] Y. Teratani, R. Sakano, R. Fujiwara, T. Hata, T. Arakawa, M. Ferrier, K. Kobayashi, and A. Oguri, J. Phys. Soc. Jpn. 85, 094718 (2016).

[37] D. R. Schmid, S. Smirnov, M. Margańska, A. Dirnaichner, P. L. Stiller, M. Grifoni, A. K. Hüttel, and C. Strunk, Phys. Rev. B 91, 155435 (2015).

[38] V. Lopes, R. A. Padilla, G. B. Martins, and E. V. Anda, Phys. Rev. B 95, 245133 (2017).

[39] C. A. Büsser and G. B. Martins, Phys. Rev. B 75, 045406 (2007).
[40] J. S. Lim, M.-S. Choi, M. Y. Choi, R. López, and R. Aguado, Phys. Rev. B 74, 205119 (2006).

[41] Y. Kleeorin and Y. Meir, Phys. Rev. B 96, 045118 (2017).

[42] P. Roura-Bas, L. Tosi, A. A. Aligia, and P. S. Cornaglia, Phys. Rev. B 86, 165106 (2012).

[43] D. Krychowski and S. Lipiński, Eur. Phys. J. B 91, 8 (2018).

[44] D. C. Langreth, Phys. Rev. 150, 516 (1966).

[45] H. Shiba, Prog. Theor. Phys. 54, 967 (1975).

[46] A. Yoshimori, Prog. Theor. Phys. 55, 67 (1976).

[47] A. C. Hewson, J. Phys.: Condens. Matter 13, 10011 (2001).

[48] A. Hewson, A. Oguri, and D. Meyer, Eur. Phys. J. B 40, 177 (2004).

[49] R. Landauer, Philos. Mag. 21, 863 (1970).

[50] Y. Meir and N. S. Wingreen, Phys. Rev. Lett. 68, 2512 (1992).

[51] S. Hershfield, J. H. Davies, and J. W. Wilkins, Phys. Rev. B 46, 7046 (1992).

[52] W. Izumida, O. Sakai, and S. Suzuki, J. Phys. Soc. Jpn. 70, 1045 (2001).

[53] A. Oguri, Phys. Rev. B 85, 155404 (2012).

[54] Y. Nishikawa, D. J. G. Crow, and A. C. Hewson, Phys. Rev. B 82, 115123 (2010).

[55] K. M. Stadler, A. K. Mitchell, J. von Delft, and A. Weichselbaum, Phys. Rev. B 93, 235101 (2016).

[56] P. Nozières, J. Low Temp. Phys. 17, 31 (1974).

[57] K. Yosida and K. Yamada, Prog. Theor. Phys. Suppl. 46, 244 (1970).

[58] K. Yamada, Prog. Theor. Phys. 53, 970 (1975).

[59] W. C. Oliveira and L. N. Oliveira, Phys. Rev. B 49, 11986 (1994).

[60] T. A. Costi, Phys. Rev. B 64, 241310(R) (2001).

[61] M. Yoshida, M. A. Whitaker, and L. N. Oliveira, Phys. Rev. B 41, 9403 (1990).

[62] R. Bulla, A. C. Hewson, and T. Pruschke, J. Phys.: Condens. Matter 10, 8365 (1998).

[63] W. Izumida, R. Okuyama, and R. Saito, Phys. Rev. B 91, 235442 (2015).

[64] W. Izumida, R. Okuyama, A. Yamakage, and R. Saito, Phys. Rev. B 93, 195442 (2016).

[65] A. C. Hewson, J. Bauer, and W. Koller, Phys. Rev. B 73, 045117 (2006).

[66] J. Bauer and A. C. Hewson, Phys. Rev. B 76, 035119 (2007).

[67] T. A. Costi, Phys. Rev. B 100, 161106(R) (2019).

[68] A. Svilans, M. Josefsson, A. M. Burke, S. Fahlvik, C. Thelander, H. Linke, and M. Leijnse, Phys. Rev. Lett. 121, 206801 (2018). 